\title{
Transcriptomic analysis reveals the gene expression profile that specifically responds to IBA during adventitious rooting in mung bean seedlings
}

Shi-Weng Li $i^{*}$, Rui-Fang Shi ${ }^{2}$, Yan Leng ${ }^{2}$ and Yuan Zhou ${ }^{2}$

\begin{abstract}
Background: Auxin plays a critical role in inducing adventitious rooting in many plants. Indole-3-butyric acid (IBA) is the most widely employed auxin for adventitious rooting. However, the molecular mechanisms by which auxin regulate the process of adventitious rooting are less well known.

Results: The RNA-Seq data analysis indicated that IBA treatment greatly increased the amount of clean reads and the amount of expressed unigenes by $24.29 \%$ and $27.42 \%$ and by $4.3 \%$ and $5.04 \%$ at two time points, respectively, and significantly increased the numbers of unigenes numbered with RPKM $=10-100$ and RPKM $=500-1000$ by $13.04 \%$ and $3.12 \%$ and by $24.66 \%$ and $108.2 \%$ at two time points, respectively. Gene Ontology (GO) enrichment analysis indicated that the enrichment of down-regulated GOs was 2.87-fold higher than that of up-regulated GOs at stage 1, suggesting that IBA significantly down-regulated gene expression at $6 \mathrm{~h}$. The GO functional category indicated that IBA significantly up- or down-regulated processes associated with auxin signaling, ribosome assembly and protein synthesis, photosynthesis, oxidoreductase activity and extracellular region, secondary cell wall biogenesis, and the cell wall during the development process. Kyoto Encyclopedia of Genes and Genomes (KEGG) pathway enrichment indicated that ribosome biogenesis, plant hormone signal transduction, pentose and glucuronate interconversions, photosynthesis, phenylpropanoid biosynthesis, sesquiterpenoid and triterpenoid biosynthesis, ribosome, cutin, flavonoid biosynthesis, and phenylalanine metabolism were the pathways most highly regulated by IBA. A total of 6369 differentially expressed (2-fold change > 2) unigenes (DEGs) with 3693 (58 \%) that were up-regulated and 2676 (42\%) down-regulated, 5433 unigenes with 2208 (40.6\%) that were up-regulated and 3225 (59.4\%) down-regulated, and 7664 unigenes with 3187 (41.6\%) that were up-regulated and 4477 (58.4\%) down-regulated were detected at stage 1, stage 2, and between stage 1 and stage 2, respectively, suggesting that IBA treatment increased the number of DEGs. A total of 143 DEGs specifically involved in plant hormone signaling and 345 transcription factor (TF) genes were also regulated by IBA. qRT-PCR validation of the 36 genes with known functions indicated a strong correlation with the RNA-Seq data.
\end{abstract}

Conclusions: The changes in GO functional categories, KEGG pathways, and global DEG profiling during adventitious rooting induced by IBA were analyzed. These results provide valuable information about the molecular traits of IBA regulation of adventitious rooting.

Keywords: Vigna radiata (L.) R.Wilczek, Adventitious root, Gene expression, Indole-3-butyric acid (IBA), Transcriptome, RNA-Seq

\footnotetext{
* Correspondence: lishweng@mail.lzjtu.cn

${ }^{1}$ School of Environmental and Municipal Engineering, Key Laboratory of

Extreme Environmental Microbial Resources and Engineering Gansu Province,

Lanzhou Jiaotong University, 88 West Anning Road, Lanzhou 730070, P. R.

China

Full list of author information is available at the end of the article
} 


\section{Background}

Adventitious roots refer to roots that form from any tissue that is not a root, such as leaves and stems. This is part of the normal development of a plant and occurs naturally or can also be induced by stresses such as wounding, flooding, and etiolation [1]. Under environmental stress, adventitious rooting acts as an alternative or supplement to seed propagation and is an important mechanism in response to stresses [2]. This method of vegetative propagation in plants has been widely used for the commercial production of woody forest and horticultural species [3]. The formation of adventitious roots has been associated with important aspects of tissue dedifferentiation and can be induced reproducibly under the control of exogenous phytohormones. Auxin acts as an effective inducer of adventitious roots in many plants and may interact with other endogenous factors or environmental stimuli during this process [4-6]. Horticultural and agricultural practices have demonstrated that the external application of auxin exerts a great effect in inducing adventitious rooting in many plant species. Indole-3-butyric acid (IBA) is used worldwide as a rooting hormone due to its higher stability and efficiency versus indole 3 -acetic acid (IAA). In cells, IBA converts into IAA and acts as a slow release source of IAA $[1,7,8]$. Auxin can induce ethylene production via the up-regulation of the 1-aminocyclopropane-1-carboxylic acid (ACC) synthase 4 (ACS4) gene, which was found to be an early auxin-induced gene $[9,10]$, to further promote adventitious rooting [11]. Although auxin plays a crucial role in inducing cell dedifferentiation and root primordium formation, the exact mechanism of its action is still poorly understood.

The physiological and biochemical changes that occur during the complex process of in vitro root development have been extensively investigated in last few decades. However, the molecular mechanisms underlying this process must be further explored. In recent years, various molecular and genetic approaches have been used to identify the genes involved in regulating adventitious root development in Arabidopsis and other plants. Among the genes identified, several gene families in the auxin signaling pathway have been shown to mediate adventitious rooting in many plants, such as the members of the auxininducible ARF (AUXIN RESPONSE FACTOR) family [12], members of the auxin-inducible GH3 (GRETCHEN $H A G E N$ 3) family [13], the tryptophan-dependent IAA biosynthesis gene YUCCA [14], the auxin efflux carrier genes PIN (PIN-FORMED) and ABCB/PGP (ATP BINDING CASSETTE-TYPE B/ P-GLYCOPROTEINS) [15-17], the auxin influx carrier gene $A U X 1 / L A X$ (AUXIN/IAA) $[15,16]$, and the auxin-responsive LOB-domain (LATERAL ORGAN BOUNDARIES-DOMAIN) genes [18]. LOB-domain transcription factors encoded by several phylogenetically, closely related genes, such as the rice genes ARL1 (ADVENTITIOUS ROOTLESS1) [19] and CRL1 (CROWN ROOTLESS1) [20], the maize gene RTCS (ROOTLESS CONCERNING CROWN AND SEMINAL ROOTS) [21], the Medicago gene MtLOB29 [22], and the Arabidopsis genes LBD16 (LATERAL ORGAN BOUNDARIES-DOMAIN) and LBD29 [23], have been identified and shown to mediate adventitious rooting. Moreover, the SCARECROW-like genes [24], the cytokinin type B response regulator (PtRR13) gene [25], and the APETALA2/ETHYLENE RESPONSE FACTOR (AP2/ERF) transcription factor genes [26] have also been demonstrated to be involved in modulating adventitious rooting. Using cDNA microarrays, Brinker et al. (2004) identified 220 genes that were significantly changed during root development in hypocotyl cuttings of Pinus contorta under IBA treatment [27]. Holmes et al. (2010) identified 904 and 993 up- and down-regulated probe sets in root-forming cultures of Medicago truncatula as well as significant changes in metabolism, signaling and the expression of transcription factors linked to in vitro adventitious root formation processes [22]. Despite this, the gene expression profile during adventitious rooting in response to auxin remains uncharacterized.

Recently, RNA-Seq has been used to explore transcriptomic data and study gene expression at the whole genome level in model and non-model organisms [28]. De novo short read assembly technology has been successfully applied to identify gene expression profiles and discover new genes without a reference genome sequence [29]. This technology platform enables the precise elucidation of transcripts present within a particular sample and can be used to calculate gene expression based on absolute transcript abundance [30]. This technology has also been used to investigate the gene expression profile during adventitious rooting. Wei et al. (2014) reported 1091 differentially expressed unigenes, including 656 upand 435 down-regulated genes, in tea cuttings (Camellia sinensis) treated with IBA. The genes involved in plant hormone signal transduction, secondary metabolism, cell wall organization, and glutathione metabolism potentially contribute to adventitious rooting [31].

Mung bean is one of the most important tropical grain legumes that serves as a significant and a cheap source of carbohydrates and easily digestible protein for the people of Asia and Africa but is also increasingly extending into Australia, USA, Canada and Ethiopia [32]. Furthermore, this plant has been widely used as a model plant species to study physiological, biochemical, and molecular mechanisms involved in the process of adventitious root formation [4, 9, 11, 33-36]. This provides an ideal experimental system with which to study the entire process of root formation, including the pre-morphogenesis stages [37]. In our previous studies, we had demonstrated that 
IBA has a great effect on promoting adventitious rooting in mung bean seedlings [38]. Recently, we had characterized the mung bean transcriptome and the differentially expressed gene profile and metabolic pathways during early stage (the induction and initiation stages) of adventitious root development in the hypocotyl cuttings without auxin treatment using RNA-Seq technology [39]. In the present study, RNA-Seq technology was exploited to highlight global changes in gene expression in response to IBA during the induction $(6 \mathrm{~h})$ and initiation $(24 \mathrm{~h})$ stages of root development in mung bean hypocotyl cuttings. We aimed to perform gene profiling, characterize the metabolic pathways specifically regulated by IBA and further to uncover the molecular basis of IBA promoting adventitious rooting. Real-time quantitative PCR was used to validate several of the transcriptional changes observed.

\section{Results and discussion}

\section{Illumina Solexa RNA paired-end sequencing and mapped} reads

To provide a comprehensive overview of mung bean and adventitious rooting in the seedlings at a transcriptional level, five cDNA libraries were constructed and sequenced from the hypocotyl tissues of 5 day-old seedlings harvested separately at $0 \mathrm{~h}$ (Con), after primary root excision and incubation in water for $6 \mathrm{~h}$ (Wat6) or $10 \mu \mathrm{M}$ IBA for $6 \mathrm{~h}$ (IBA6), and after primary root excision and incubation in water for $24 \mathrm{~h}$ (Wat24) or IBA for $24 \mathrm{~h}$ (IBA24) (Fig. 1), of which, the Con, Wat6, and Wat24 were previously sequenced in our previous study [39]. The sequencing data and mapping results of the cDNA libraries of IBA6 and IBA24 were listed in Additional file 1 and Table 1 . On average, $94 \%$ of the quality filter passed reads generated for all five samples was mapped uniquely to the reference sequences. Data analysis showed that the amounts of raw reads and clean reads were increased by $24.28 \%$ and $24.29 \%$ at stage 1 , respectively, and increased by $23.93 \%$ and $27.42 \%$ at stage 2, respectively, reflecting a great increase in the number of sequences resulting from IBA treatment.

\section{De novo assembly, unigene determination and abundance measurement}

The paired-end de novo assembly of the processed reads was performed using the TRINITY transcriptome assembly software program, which can recover more full-length transcripts across a broad range of expression levels and provides a unified, sensitive solution for transcriptome reconstruction in species without a reference genome, similar to methods that rely on genome alignments [40]. The Chrysalis cluster module of TRINITY was used to cluster the transcripts and generated a total of 78,697 unigenes [39]. The ratios of expressed unigenes were $84.71 \%$ (66,663), $88.35 \%(69,527), 82.19 \%(64,680)$, and $86.33 \%$ $(67,938)$ in Wat6, IBA6, Wat24, and IBA24, respectively. These results indicate that the amounts of unigenes expressed at stage 1 and stage 2 were increased by $4.3 \%$ (2864 unigenes) and $5.04 \%$ (3258 unigenes), respectively. Clearly, IBA enhanced the number of genes expressed during the early stages of rooting (Table 1 ).

To evaluate the abundances of the expressed unigenes, gene expression levels were estimated from Illumina sequencing based on the number of clean reads for a gene. The RPKM method [30] was used to calculate the expression abundances of unigenes in each sample. The results showed that the unigenes numbered with RPKM $=10-100$ were significantly increased by $13.04 \%$ at stage 1 and by $3.12 \%$ at stage 2 . The unigenes numbered with RPKM = $500-1000$ were significantly increased by $24.66 \%$ at stage 1 and by $108.2 \%$ at stage 2 . These results suggest that the expression abundances of certain genes were greatly increased by IBA treatment (Table 1).

\section{GO enrichment analysis}

To discern global patterns of differential transcript abundance over the time course, unigenes with contrasting

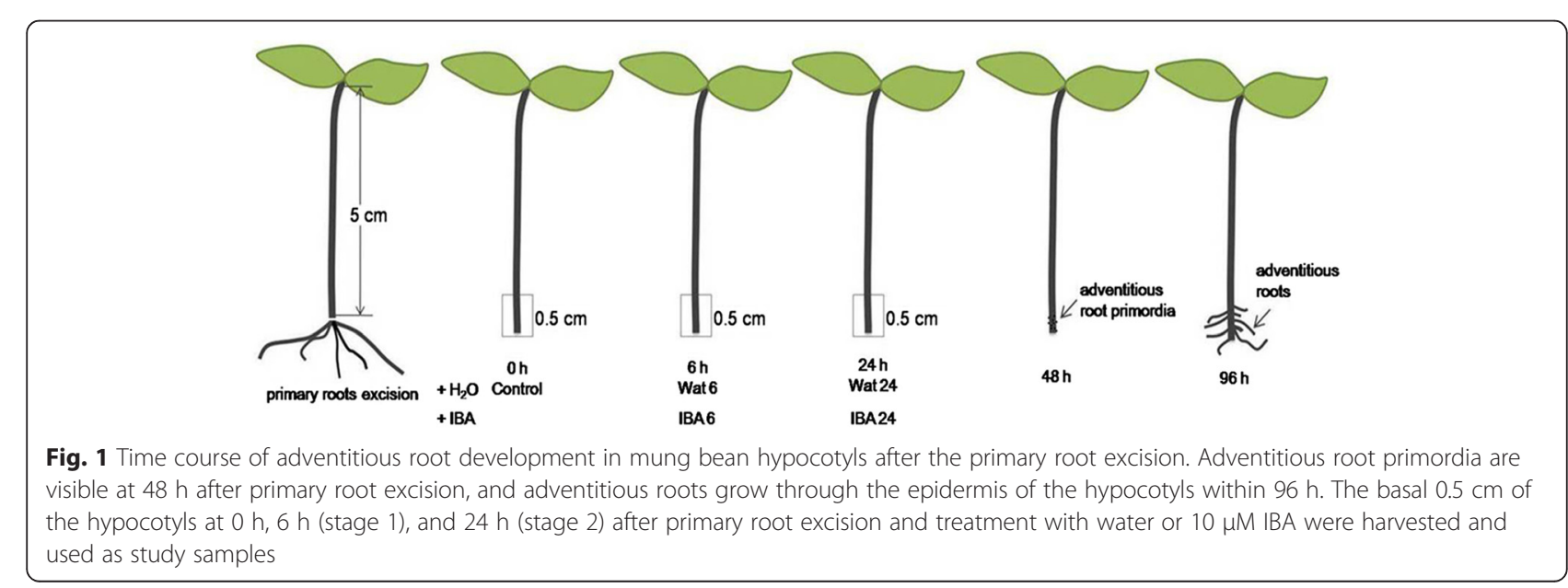


Table 1 Sample mapping results and unigene abundance measurements in the samples

\begin{tabular}{lll}
\hline & IBA6 & IBA24 \\
\hline Clean reads & 69082097 & 70805846 \\
Mapped reads & 64711415 & 66557328 \\
Mapped ratio & $93.67 \%$ & $94.00 \%$ \\
All unigenes & 78697 & 78697 \\
Expressed unigenes & 69527 & 67938 \\
Expressed ratio & $88.35 \%$ & $86.33 \%$ \\
RPKM $\geq 1000$ unigenes & 41 & 46 \\
RPKM 500-1000 unigenes & 91 & 127 \\
RPKM 100-500 unigenes & 957 & 976 \\
RPKM 10-100 unigenes & 9824 & 9613 \\
RPKM 1-10 unigenes & 15535 & 14053 \\
RPKM <1 unigenes & 43076 & 43120 \\
Maxim RPKM & 24455 & 9746 \\
Average RPKM & 10.08 & 10.44 \\
\hline
\end{tabular}

significance at FDR $<0.05$ were further filtered to include only those with greater than 2 -fold changes $(\log 2>1)$ in unigene abundance between the samples. Unigenes meeting these criteria were clustered using Blast2GO software (version 2.3.5, http://www.blast2go.de/) [41] and WEGO software [42]. To evaluate the GOs differentially regulated during the time course, the significantly up- and downregulated GOs were enriched between IBA6 and Con and between IBA24 and Con. The results showed that the upand down-regulated enriched GOs decreased by $63.7 \%$ (379 GOs) and $5.6 \%$ (17 GOs) at stage 1, respectively, and increased by $14.7 \%$ (34 GOs) and $16.1 \%$ (41 GOs) at stage 2, respectively. Furthermore, the up- and downregulated enriched GOs decreased by $34.3 \%$ (82 GOs) and $71.2 \%$ (489 GOs), respectively, from stage 1 to stage 2. The down-regulated enriched GOs were 2.87-fold (448 GOs) higher than that of the up-regulated at stage 1, while the down-regulated enriched GOs were increased by $26.1 \%$ (41 GOs) compared to the up-regulated GOs at stage 2 (Fig. 2). These results indicate that the majority of GOs were significantly down-regulated at stage 1, suggesting IBA treatment significantly down-regulated gene expression after $6 \mathrm{~h}$ of adventitious rooting. The results further suggest that profound cellular and metabolic reorganization occurs with 6 h of rooting.

The differentially regulated GO subcategories are presented in Figs. 3 and 4. In the group of up-regulated GO terms, the percentages of differentially expressed genes (DEGs) in most of the GO subcategories at stage 1 were higher than those at stage 2 with the exceptions of nutrient reservoir activity and structural molecule activity in molecular function, suggesting that most of the DEGs were significantly up-regulated at $6 \mathrm{~h}$ after IBA treatment.

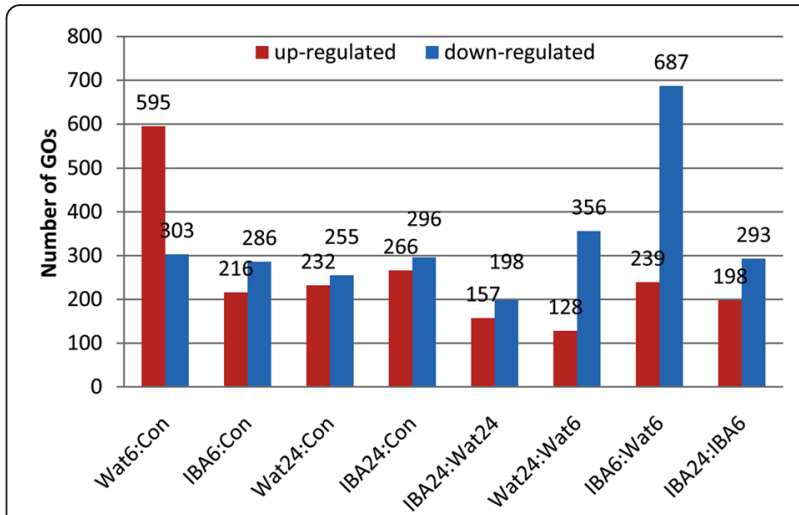

Fig. 2 The distribution of GO terms enriched in the sample pairs

Furthermore, the percentage of DEGs clustered into the categories of rhythmic process in biological process, extracellular region and extracellular region part in cellular component, and antioxidant activity, electron carrier activity, enzyme regulator activity, and nutrient reservoir activity in molecular function were strongly increased from stage 1 to stage 2 . In the group of down-regulated GO terms, nearly equal percentages of DEGs in most of the subcategories were examined between stage 1 and stage 2, with the exception of cell killing in biological process, extracellular matrix, extracellular region, and extracellular region part in cellular component, nutrient reservoir activity, and structural molecule activity in molecular function. In the subcategories of cell killing, extracellular matrix, extracellular region, and extracellular region part, the DEGs ratio at stage 1 was higher than that at stage 2, whereas for nutrient reservoir activity and structural molecule activity, the DEGs ratio at stage 1 was lower than that at stage 2. These results suggest that profound changes in biological function caused by IBA occurred in GO subcategories such as extracellular region, extracellular matrix, extracellular region part, antioxidant activity, electron carrier activity, cell killing, and structural molecule activity during early stages of IBA-induced rooting.

Additional file 2 and Table 2 list the top 50 significantly up- and down-regulated GO categories during the IBA-induced development process. The results revealed that the GOs associated with auxin signaling, such as auxin mediated signaling pathway, cellular response to auxin stimulus, hormone-mediated signaling pathway, response to auxin stimulus, cellular response to hormone stimulus, and hormone-mediated signaling pathway, were significantly up-regulated both at stage 1 and stage 2. The GOs associated with ribosome assembly and protein synthesis, such as nucleolus, ribonucleoprotein complex biogenesis, ribosome biogenesis, rRNA metabolic process, rRNA processing, ribosome, structural constituent of ribosome, ribosomal subunit, cytosolic ribosome, and translation, were significantly up-regulated at 


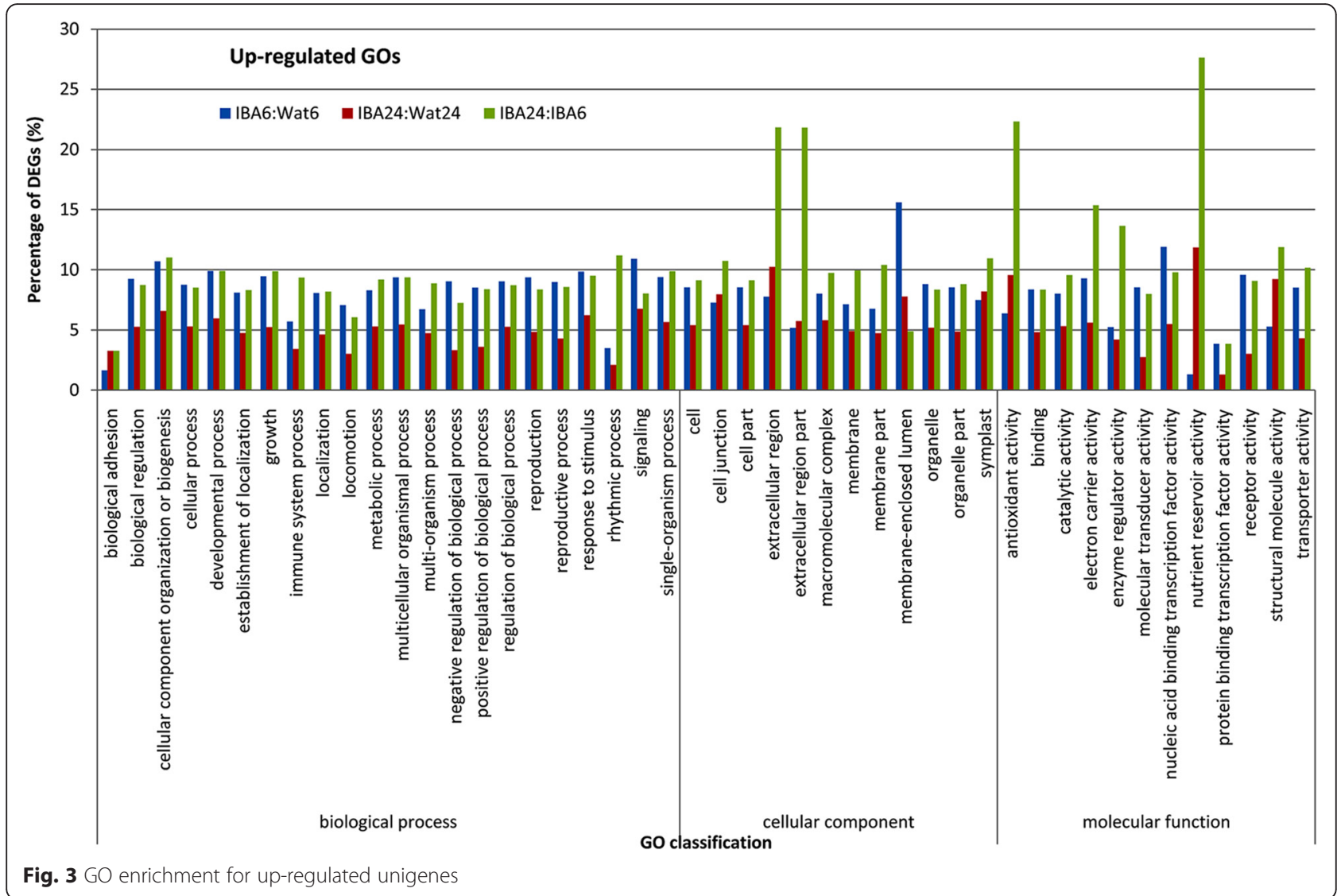

stage 1 but significantly down-regulated at stage 2 . The GOs associated with photosynthesis, such as photosystem, photosynthesis, photosystem II, and photosystem I, were significantly down-regulated at stage 1 . The GOs for oxidoreductase activity and extracellular region were significantly down-regulated both at stage 1 and stage 2 . The GO secondary cell wall biogenesis was down-regulated at stage 1 , while the GO cell wall was up-regulated at stage 2. The GOs associated with photosynthesis, such as light reaction, photosystem, photosystem II, and thylakoid, as well as cell wall, external encapsulating structure, and hydrolase activity (hydrolyzing O-glycosyl compounds), were significantly up-regulated from stage 1 to stage 2 . However, the GOs associated with hormone pathway and signaling such as response to chemical stimulus, response to hormone stimulus, hormone-mediated signaling pathway, response to organic substance, cellular response to hormone stimulus, response to auxin stimulus, cellular response to endogenous stimulus, nucleic acid binding transcription factor activity, and sequence-specific DNA binding transcription factor activity, were significantly downregulated from stage 1 to stage 2 .

\section{KEGG pathway enrichment analysis}

To further determine which biological pathways were significantly (FDR $\leq 0.05)$ modulated during the process, KEGG pathway enrichment was performed using the KEGG Automatic Annotation Server (KAAS) [43]. This analysis revealed that 12 and five pathways were enriched at stage 1 and stage 2, respectively. Among these, five KOs were significantly down-regulated and two KOs were significantly up-regulated at stage 1, suggesting that significant metabolic changes occur at the 6-h time point (Table 3, Additional file 3). Further analysis indicated that the pathways of ribosome biogenesis in eukaryotes and plant hormone signal transduction were up-regulated both at stage 1 and stage 2 , and the pathway of pentose and glucuronate interconversions, which has a function in glucuronate and pectin degradation, was up-regulated at stage 2 . The significantly down-regulated KOs at stage 1 included photosynthesis, photosynthesis-antenna proteins, phenylpropanoid biosynthesis, and sesquiterpenoid and triterpenoid biosynthesis, while those at stage 2 were ribosome, phenylpropanoid biosynthesis, cutin, flavonoid biosynthesis, and phenylalanine metabolism. When compared with stage 1, photosynthesis, phenylpropanoid biosynthesis, starch and sucrose 


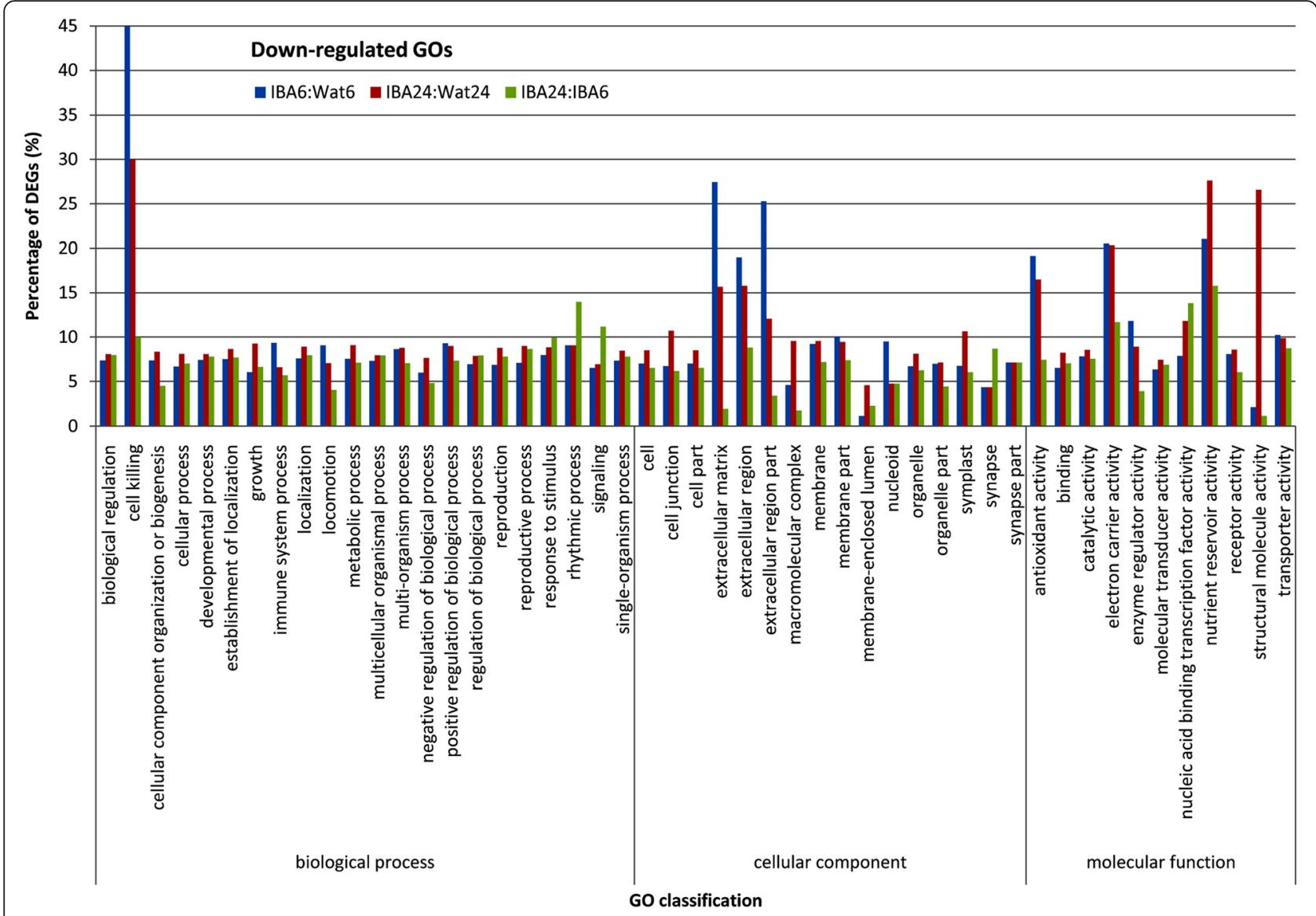

Fig. $4 \mathrm{GO}$ enrichment for down-regulated unigenes

metabolism, cell cycle, and ribosome were up-regulated, while plant hormone signal transduction, diterpenoid biosynthesis, aminobenzoate degradation, bisphenol degradation, glucosinolate biosynthesis, and phenylalanine metabolism were down-regulated from stage 1 to stage 2 . The phenylpropanoid biosynthesis pathway is required for suberin and lignin biosynthesis. The cutin pathway is involved in suberin and wax biosynthesis. Flavonoids are a major class of plant secondary metabolites that serve multitude of functions, including as pigments and in antioxidant activity. The pathways of aminobenzoate degradation and bisphenol degradation have functions in xenobiotics biodegradation and metabolism. These results indicate that the plant hormone signal transduction and ribosome biogenesis pathways were significantly up-regulated by IBA treatment, photosynthesis activity was down-regulated at stage 1 but up-regulated at stage 2 , and plant hormone signal transduction was down-regulated from stage 1 to stage 2. Furthermore, the pectin degradation pathway was up-regulated and suberin, lignin and wax biosynthesis were downregulated at both stage 1 and stage 2 , suggesting the beginning of cell wall loosening from stage 1 to stage 2 .
The secondary metabolism pathways, such as phenylpropanoids and associated flavonoids, might play important roles in IBA-induced adventitious rooting. Phenylpropanoids function in plant defense as inducible chemical barriers or as signaling molecules. The core phenylpropanoid pathway is organized from phenylalanine to an activated (hydroxy) cinnamic acid derivative, and the specific branch pathways lead to the formation of lignin, sinapate esters, condensed tannins, anthocyanins, coumarins, benzoic acids and flavonoids/ isoflavonoids [44-46]. The common derivatives from the phenylpropanoid pathway, specifically phenolic acids, flavonoids, and lignin [47], are crucial regulators in cell division and differentiation [48] and stimulate in vitro rooting [49].

Global changes in differentially expressed genes (DEGs) during adventitious rooting in response to IBA

To examine a gene expression profile during rooting under IBA treatment, the clean reads for a gene were mapped back to the assembled unigenes using BWA0.6.2 software. The number of reads mapped to each unigene was then counted and normalized using RPKM [50]. Gene expression values were measured using the 
Table 2 Top-5 significantly up- and down-regulated GOs between the samples

\begin{tabular}{|c|c|c|c|c|c|}
\hline GO ID & Total genes & DEGs & FDR & Level & Terms \\
\hline \multicolumn{6}{|l|}{ IBA6:Wat6 } \\
\hline \multicolumn{6}{|c|}{ Down-regulated } \\
\hline GO:0005576 & 1222 & 232 & 5.87E-38 & 2 & extracellular region \\
\hline GO:0016491 & 1856 & 291 & $1.34 \mathrm{E}-32$ & 3 & oxidoreductase activity \\
\hline GO:0009521 & 79 & 49 & $3.74 \mathrm{E}-32$ & 3 & photosystem \\
\hline GO:0015979 & 192 & 72 & $3.66 \mathrm{E}-29$ & 4 & photosynthesis \\
\hline GO:0046906 & 485 & 119 & 7.40E-29 & 4 & tetrapyrrole binding \\
\hline \multicolumn{6}{|l|}{ Up-regulated } \\
\hline GO:0005730 & 378 & 103 & $1.06 \mathrm{E}-24$ & 5 & nucleolus \\
\hline GO:0022613 & 200 & 70 & $2.29 \mathrm{E}-23$ & 4 & ribonucleoprotein complex biogenesis \\
\hline GO:0042254 & 186 & 67 & $2.73 \mathrm{E}-23$ & 5 & ribosome biogenesis \\
\hline GO:0009734 & 238 & 72 & 4.81E-20 & 6 & auxin mediated signaling pathway \\
\hline GO:0071365 & 247 & 72 & 4.35E-19 & 6 & cellular response to auxin stimulus \\
\hline \multicolumn{6}{|l|}{ IBA24:Wat24 } \\
\hline \multicolumn{6}{|c|}{ Down-regulated } \\
\hline GO:0005840 & 538 & 161 & $2.51 \mathrm{E}-42$ & 4 & ribosome \\
\hline GO:0003735 & 471 & 147 & 4.33E-41 & 3 & structural constituent of ribosome \\
\hline GO:0005198 & 606 & 161 & $1.42 \mathrm{E}-35$ & 2 & structural molecule activity \\
\hline GO:0006412 & 671 & 157 & $8.65 \mathrm{E}-28$ & 6 & translation \\
\hline GO:0030529 & 801 & 165 & $9.85 \mathrm{E}-23$ & 3 & ribonucleoprotein complex \\
\hline \multicolumn{6}{|l|}{ Up-regulated } \\
\hline GO:0009734 & 238 & 54 & $6.20 \mathrm{E}-17$ & 6 & auxin mediated signaling pathway \\
\hline GO:0071365 & 247 & 55 & $6.20 \mathrm{E}-17$ & 6 & cellular response to auxin stimulus \\
\hline GO:0009733 & 367 & 68 & 7.03E-17 & 5 & response to auxin stimulus \\
\hline GO:0005618 & 654 & 87 & $1.06 \mathrm{E}-12$ & 4 & cell wall \\
\hline GO:0030312 & 671 & 87 & $3.91 \mathrm{E}-12$ & 3 & external encapsulating structure \\
\hline \multicolumn{6}{|l|}{ IBA24:IBA6 } \\
\hline \multicolumn{6}{|c|}{ Down-regualted } \\
\hline GO:0042221 & 2287 & 297 & $6.01 \mathrm{E}-21$ & 3 & response to chemical stimulus \\
\hline GO:0009719 & 1364 & 204 & $1.28 \mathrm{E}-20$ & 3 & response to endogenous stimulus \\
\hline GO:0009725 & 1235 & 182 & $1.63 \mathrm{E}-17$ & 4 & response to hormone stimulus \\
\hline GO:0009755 & 768 & 130 & $5.55 \mathrm{E}-17$ & 5 & hormone-mediated signaling pathway \\
\hline GO:0010033 & 1584 & 215 & $5.55 \mathrm{E}-17$ & 4 & response to organic substance \\
\hline \multicolumn{6}{|l|}{ Up-regulated } \\
\hline GO:0005576 & 1222 & 267 & $5.64 \mathrm{E}-39$ & 2 & extracellular region \\
\hline GO:0015979 & 192 & 65 & $6.90 \mathrm{E}-18$ & 4 & photosynthesis \\
\hline GO:0005618 & 654 & 133 & $2.56 \mathrm{E}-15$ & 4 & cell wall \\
\hline GO:0030312 & 671 & 134 & 7.04E-15 & 3 & external encapsulating structure \\
\hline GO:0019684 & 82 & 34 & 7.93E-12 & 5 & photosynthesis, light reaction \\
\hline
\end{tabular}

method described by DEGseq R package [51]. Of these, 6369 unigenes specifically with 3693 (58 \%) up-regulated and 2676 (42\%) down-regulated, 5433 unigenes with 2208 (40.6 \%) up-regulated and 3225 (59.4 \%) downregulated, and 7664 unigenes with 3187 (41.6 \%) up- regulated and 4477 (58.4\%) down-regulated were differentially expressed $(\log 2 \geq 1)$ at stage 1 , stage 2 , and between stage 1 and stage 2, respectively. When compared with the control (Con), the number of DEGs $(\log 2 \geq 1)$ exhibited the following trend: IBA24 $>$ IBA6 $>$ Wat24 $>$ 
Table 3 Top most significantly up- and down-regulated KOs between the samples

\begin{tabular}{|c|c|c|c|c|}
\hline $\mathrm{KO}$ ID & Total genes & DEGs & $\mathrm{FDR} \leq 0.05$ & Description \\
\hline \multicolumn{5}{|l|}{ IBA6:Wat6 } \\
\hline \multicolumn{5}{|c|}{ Down-regulated } \\
\hline ko00195 & 53 & 31 & $1.29 \mathrm{E}-20$ & Photosynthesis \\
\hline ko00196 & 15 & 12 & $5.01 \mathrm{E}-10$ & Photosynthesis- antenna proteins \\
\hline ko00940 & 69 & 18 & 5.25E-05 & Phenylpropanoid biosynthesis \\
\hline ko00909 & 7 & 5 & 0.0013 & Sesquiterpenoid and triterpenoid biosynthesis \\
\hline \multicolumn{5}{|c|}{ Up-regulated } \\
\hline ko03008 & 73 & 32 & $1.20 \mathrm{E}-14$ & Ribosome biogenesis in eukaryotes \\
\hline ko04075 & 130 & 20 & 0.0256 & Plant hormone signal transduction \\
\hline \multicolumn{5}{|c|}{ IBA24:Wat24 } \\
\hline \multicolumn{5}{|c|}{ Down-regulaed } \\
\hline ko03010 & 337 & 115 & $1.37 \mathrm{E}-41$ & Ribosome \\
\hline ko00940 & 69 & 18 & 0.0016 & Phenylpropanoid biosynthesis \\
\hline ko00073 & 12 & 6 & 0.0176 & Cutin \\
\hline ko00941 & 17 & 7 & 0.0176 & Flavonoid biosynthesis \\
\hline ko00360 & 57 & 13 & 0.0408 & Phenylalanine metabolism \\
\hline \multicolumn{5}{|c|}{ Up-regulated } \\
\hline ko03008 & 73 & 17 & 5.09E-06 & Ribosome biogenesis in eukaryotes \\
\hline ko03010 & 337 & 38 & 2.91E-05 & Ribosome \\
\hline ko04075 & 130 & 18 & 0.0020 & Plant hormone signal transduction \\
\hline ko00040 & 49 & 9 & 0.0164 & Pentose and glucuronate interconversions \\
\hline \multicolumn{5}{|c|}{ IBA24:IBA6 } \\
\hline \multicolumn{5}{|c|}{ Down-regulated } \\
\hline ko04075 & 130 & 24 & 0.0020 & Plant hormone signal transduction \\
\hline ko00904 & 8 & 5 & 0.0083 & Diterpenoid biosynthesis \\
\hline ko00627 & 29 & 9 & 0.0084 & Aminobenzoate degradation \\
\hline ko00363 & 20 & 7 & 0.0154 & Bisphenol degradation \\
\hline ko00966 & 6 & 4 & 0.0154 & Glucosinolate biosynthesis \\
\hline \multicolumn{5}{|c|}{ Up-regulated } \\
\hline ko00195 & 53 & 19 & 5.30E-06 & Photosynthesis \\
\hline ko00940 & 69 & 17 & 0.0033 & Phenylpropanoid biosynthesis \\
\hline ko00500 & 119 & 23 & 0.0067 & Starch and sucrose metabolism \\
\hline ko04110 & 94 & 19 & 0.0117 & Cell cycle \\
\hline ko03010 & 337 & 47 & 0.0142 & Ribosome \\
\hline
\end{tabular}

Wat6. The DEGs number was increased by $22.7 \%$ at stage 1 and by $40.3 \%$ at stage 2 . Clearly, IBA treatment increased the number of DEGs. Moreover, the downregulated DEGs accounted for $75.1 \%, 65.8 \%, 73.0 \%$, and $72.2 \%$ in Wat6, IBA6, Wat24, and IBA24, respectively, indicating the dominance of significantly downregulated DEGs during the adventitious rooting process. The up-regulated DEGs accounted for $58.0 \%$ and $40.6 \%$ at stage 1 and stage 2 , respectively, and total DEGs was increased by $17.2 \%$ in IBA6 compared with IBA24. This result indicates that IBA treatment significantly up-regulated gene expression, and the greatest changes in gene expression occurred at the 6-h time point (Fig. 5 and Table 4). Further analysis revealed that the DEGs with $2>\log 2 \geq 1$ accounted for $62.8 \%$ and $60.8 \%$ at stage 1 and stage 2, respectively, and the DEGs with $\log 2 \geq 5$ accounted for $1.4 \%$ and $1.5 \%$ at stage 1 and stage 2 , respectively. This indicates that the DEGs with 2- to 4-fold changes accounted for more than $60 \%$ of the total, and there was no correlation between DEGs number and the time of IBA treatment (Table 4). 


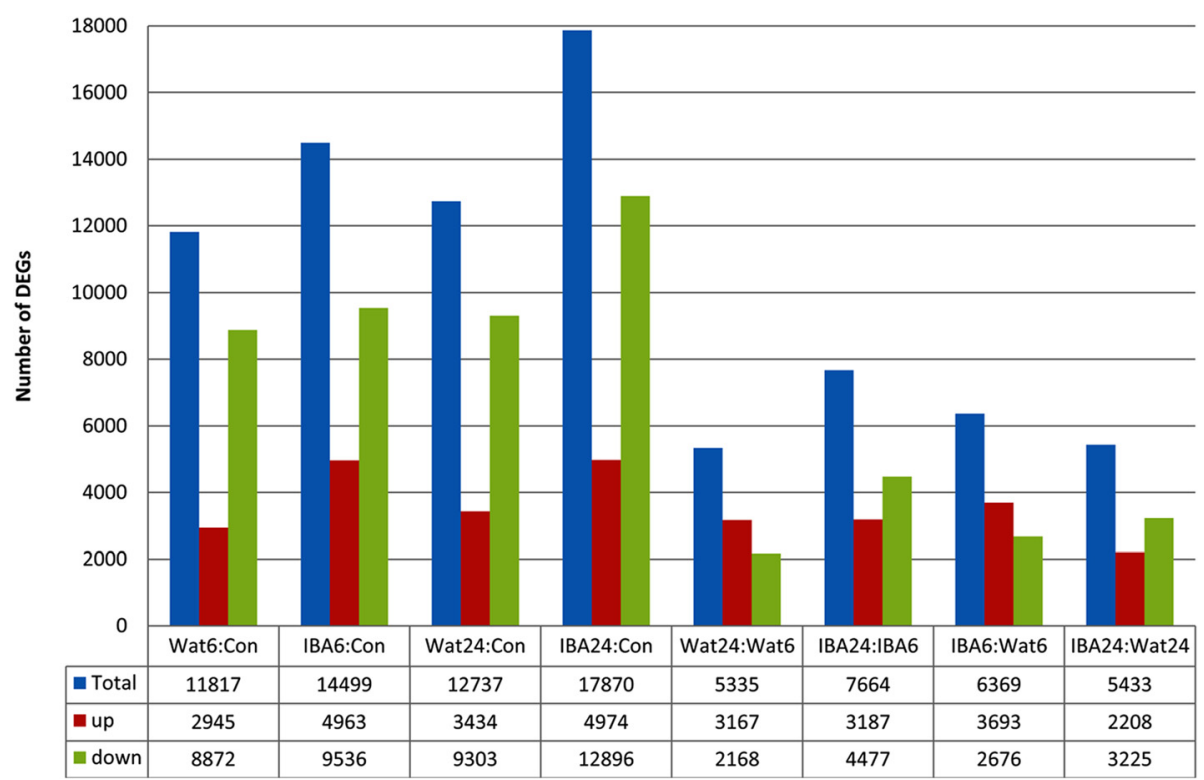

Fig. 5 The distribution of DEGs in the sample pairs

\section{Gene expression patterns in response to IBA during the} early stages of adventitious rooting

To gain insight into the gene expression patterns that occur in response to IBA during adventitious rooting, transcript levels of annotated unigenes were compared between different developmental time-points as well as between treatments. After filtering out the unigenes designated as unknown, hypothetical protein, uncharacterized protein, predicted protein, and unnamed protein in the database, the most significantly and abundantly expressed DEGs with both RPKM $>10$ and $\log 2>3$ were selected and are listed in Additional files 4, 5 and 6.

Among the most significantly and abundantly expressed unigenes at stage 1, 17 DEGs with RPKM >10 were upregulated 16- to 82-fold and 41 DEGs with RPKM $>10$ were up-regulated 8- to 16-fold. Thirty seven DEGs with RPKM > 10 were down-regulated 16- to 222-fold and 23 unigenes with RPKM >10 were down-regulated 8- to 16fold. Of those at stage 2, 13 DEGs with RPKM > 10 were

Table 4 Statistics of the DEGs between the time points during adventitious rooting

\begin{tabular}{llllllllll}
\hline Samples & \multicolumn{1}{c}{ Log2 } & & & & & & & Total \\
\cline { 3 - 8 } & & Total & NA & $\geq 5$ & $\geq 4$ & $\geq 3$ & $\geq 2$ & $\geq 1$ & DEGs \\
\hline IBA6:Wat6 & down & 2676 & 177 & 60 & 116 & 249 & 551 & 1523 & 6369 \\
& up & 3693 & 169 & 32 & 95 & 265 & 653 & 2479 & \\
IBA24:Wat24 & down & 3225 & 239 & 56 & 132 & 345 & 627 & 1826 & 5433 \\
& up & 2208 & 139 & 24 & 64 & 163 & 340 & 1478 & \\
IBA24:IBA6 & down & 4477 & 356 & 73 & 173 & 447 & 1051 & 2377 & 7664 \\
& up & 3187 & 293 & 65 & 116 & 308 & 698 & 1707 & \\
\hline
\end{tabular}

up-regulated 16- to 63-fold and 23 DEGs with RPKM > 10 were up-regulated 8- to 16-fold. Twenty three DEGs with RPKM > 10 were down-regulated 16- to 388-fold, and 31 DEGs with RPKM > 10 were down-regulated 8- to 16fold. The changes in DEGs between stage 1 and stage 2 were further analyzed. Thirty two DEGs with RPKM >10 were up-regulated 16- to 603-fold and 41 DEGs with RPKM >10 were up-regulated 8- to 16-fold at stage 2 . Thirty eight DEGs with RPKM $>10$ were down-regulated 16 - to 89-fold and 41 DEGs were down-regulated 8- to 16 -fold at stage 2 . These DEGs can be clustered into 11 functional categories as detailed below.

\section{Auxin homeostasis and signaling}

During stage 1, 17 genes including five IAA-amido synthetase GH3 genes (Vr39322, Vr42095, Vr13365, Vr41585, and Vr13365), ten auxin-induced protein genes, and two auxin-responsive protein genes (Vr44933 and Vr16403) were strongly up-regulated; only an IAA-amino acid hydrolase ILR1-like and an auxin-induced protein 5NG4like gene were down-regulated. During stage 2, 11 genes including five GH3 genes, four auxin-induced protein genes, an auxin-responsive protein IAA12-like gene, and an auxin-binding protein ABP19a-like gene were upregulated; the hydrolase activity gene was down-regulated. When compared with stage 1, the gene encoding for auxin-binding protein ABP19a-like (RPKM =52.8) was specifically up-regulated at stage 2 . Sixteen auxin-induced protein genes were down-regulated. The GH3 gene family encodes IAA-amido synthetases that negatively regulate the levels of free IAA by conjugating IAA to amino acids. IAA-amino acid hydrolase hydrolyzes the amide bond 
between IAA and the conjugated amino acid to release active IAA. IAA-amino acid hydrolase coordinates with PIN and GH3s to maintain auxin homeostasis in the adventitious roots [7]. The IBA-induced overexpression of the GH3 genes and the IBA-mediated repression of the IAAamino acid hydrolase gene both in stage 1 and stage 2 suggest a reduction of free auxin and an increase in amino-IAA during the early stages of adventitious rooting. The auxin-induced overexpression of the GH3-3 gene was also investigated during the initiation of adventitious roots in both the hypocotyls and stems of Arabidopsis [52]. In addition, GH3s also control jasmonic acid (JA) homeostasis by conjugating JA to amino acids. Auxin controls JA homeostasis and stimulates adventitious rooting by inducing GH3 expression, leading to an increase in JA conjugation and a reduction in free JA levels. This signaling pathway fine-tunes adventitious root initiation in Arabidopsis hypocotyls [13]. An additional auxin receptor, ABP1 (AUXIN BINDING PROTEIN1), rapidly mediates cellular auxin effects via a non-transcriptional auxin response pathway and is essential for early auxin responses [53]. The specifically up-regulated ABP19a-like gene indicates that the mediation of $\mathrm{ABP}$ in rapid auxin signaling occurred in stage 2. Moreover, genes encoding auxininduced proteins were down-regulated in stage 2, which may result in the initiation of auxin signaling due to the derepression of ARFs during stage 2.

\section{Ethylene pathway-related DEGs}

During stage 1, eight genes, including three 1aminocyclopropane-1-carboxylic acid (ACC) synthase (ACS) genes (Vr45385, Vr10942, and Vr10988), three ethylene-responsive transcription factor 1B-like genes (Vr28889, Vr42199, and Vr30921), a S-adenosylmethionine (SAM) decarboxylase proenzyme-like gene, and a L,L-diaminopimelate aminotransferase gene, were up-regulated; an ACC oxidase (ACO) homolog 1-like gene was downregulated. No DEGs were selected with the setting criteria during stage 2 . When compared with stage 1 , the genes for an ethylene-responsive transcription factor LEP-like and an ethylene receptor were up-regulated; genes coding for an ACS, two ACO (Vr43821 and Vr44578), two ethyleneresponsive transcription factor 15-like (Vr31499 and Vr52928), and a L,L-diaminopimelate aminotransferase were down-regulated. S-adenosylmethionine decarboxylase and L,L-diaminopimelate aminotransferase are involved in ethylene biosynthesis [54]. In the ethylene biosynthetic pathway, ACS converts SAM to ACC, and then ACO converts ACC to ethylene [54]. This result indicates that IBA significantly up-regulates the expression of $A C S$ and subsequently promotes ethylene biosynthesis $[6,55]$. Ethylene biosynthesis and perception and ethylene-auxin crosstalk are required for adventitious root formation [56]. Auxin promotes ethylene biosynthesis by up-regulating the expression of several $A C S$ genes [54], thereby increasing ethylene levels [56]. Ethylene positively regulates IAA synthesis and promotes polar auxin transport and accumulation via AUX1 [55-57].

\section{Cytokinin pathway-related DEGs}

Several genes involved in the cytokinin biosynthesis pathway, including two cytokinin riboside 5'-monophosphate phosphoribohydrolase genes (Vr51879 and Vr25044) and a cytokinin dehydrogenase 3-like gene, were down-regulated during stage 2 compared with stage 1. Cytokinin has been known to be a negative regulator of adventitious rooting via its negative regulation of auxin [25]. IBA-induced adventitious rooting might be related to the inhibition of cytokinin synthesis by IBA in this study.

\section{Transcription factors}

During stage 1, nine genes including a LOB29 gene, a LOB16 gene, two protein SOMBRERO-like genes (Vr44306 and Vr49314), three homeobox-leucine zipper protein ATHB-like genes (Vr74958, Vr21565 and Vr33455), a WRKY (WRKYGQK domain) 72-like gene, and a bHLH (basic/helix-loop-helix) 135-like gene were highly up-regulated; six genes including a MYB1(avian myeloblastosis viral oncogene homolog) 13-like gene, a MYB-related protein 308-like gene, a bHLH18-like gene, a transcription factor CPC gene, and a LOB 21-like gene were down-regulated. During stage 2, genes encoding $\mathrm{LOB}$ domain proteins such as LOB 12-like, LOB 16-like, LOB 18-like, and LOB 29-like were up-regulated; a MYB113-like gene was down-regulated. When compared with stage 1 , genes coding for a SCR (SCARECROW)-like protein 28like and a MADS-box transcription factor 1-like were upregulated, and genes coding for bHLH137-like, BEE 3-like, and NAC (NAM, ATAF, and CUC2) 72-like were downregulated at stage 2. SOMBRERO-like genes encoding NAM (NO APICAL MERISTEM) family proteins modulate meristems and primordial development and promote the expression of genes involved in secondary cell walls biosynthesis [58]. SCR genes in the GRAS family are known to be involved in meristem maintenance and root meristem initiation in adventitious root primordia $[24,59$, $60]$. Interestingly, the expression of these genes is induced by exogenous auxin in the rooting-competent cuttings of two distantly related forest species, Pinus radiata and Castanea sativa, during the early stage leading to adventitious root formation [24].

\section{Cell wall modification}

During stage 1, seven genes including two expansin precursor genes (Vr38364 and Vr49341), two UDPglucosyltransferase genes (Vr236166 and Vr36946), a 
nonsymbiotic hemoglobin gene, a cationic peroxidase 1-like gene, and a cytokinin induced message gene were up-regulated; 15 genes including three genes of fasciclin-like arabinogalactan protein (Vr36181, Vr22704, and Vr40615), five genes encoding cellulose synthase (Vr46819, Vr44425, Vr43928, Vr41179, and Vr38347), three genes encoding glycosyl transferase family protein (Vr39089, Vr36076, and Vr48851), a COBRA-like protein gene, an organ-specific protein S2-like gene, a lectin gene, and a polygalacturonase non-catalytic subunit JP650-like gene were down-regulated. Expansins have been demonstrated to be induced in hypocotyl bases during the early stages of adventitious root induction in Pinus taeda and function in the loosening of cellulose and hemicellulose [61]. The cytokinin-induced message protein, which belongs to expansin family protein, is considered to be involved in cell wall loosening [58]. Cationic peroxidase has been shown to be involved in the biosynthesis of lignin and suberin [62]. Polygalacturonase is one of the pectin lyase associated with the degradation of the pectin network in the plant cell wall [58]. The ortholog of fasciclinlike arabinogalactan protein of Arabidopsis may be a cell surface adhesion protein that is required for normal cell expansion and is involved in cell wall biogenesis and organization [58]. COBRA-like protein is involved in cellulose microfibril organization [58]. Therefore, our results indicate that the process of cell wall loosening was significantly up-regulated, whereas the process of cell wall synthesis was suppressed by IBA after $6 \mathrm{~h}$ of treatment.

During stage 2, 10 genes associated with cell wall loosening including two expansin genes (Vr55545 and Vr38364), two protein SOMBRERO-like genes (Vr44306 and Vr49314), a galactoside 2-alpha-L-fucosyltransferase-like, a polygalacturonase-like, a pectate lyase 8-like, a cytokinin-induced message, two cationic peroxidase 1like genes (Vr43362 and Vr46509), and a BURP domaincontaining protein 3-like were up-regulated; 17 genes associated with cell wall synthesis including six cellulose synthase A catalytic subunit 7 genes (Vr54568, Vr43928, Vr41179, Vr44425, Vr46089, and Vr46819), four genes encoding casparian strip membrane protein (CASP) (Vr36698, Vr68124, Vr79590, and Vr77808), three fasciclin-like arabinogalactan protein genes (Vr40615, Vr37484, and Vr36181), a xylosyltransferase 1-like gene, a lectin gene, an organ-specific protein S2-like gene, a xyloglucan endotransglucosylase/hydrolase protein 32like genes, a UDP-galactose transporter 2-like gene, a COBRA-like protein 4-like gene, a laccase-3-like gene, and a mannan endo-1,4-beta-mannosidase 4-like gene were down-regulated during stage 2 .

When compared with stage 1 , putative expansin-A17like (RPKM =43.6) was specifically up-regulated at stage 2. Seventeen genes including three polygalacturonase PG1 genes (Vr39755, Vr41762, and Vr44288), two endoglucanase (Vr34411 and Vr53145), a galactoside 2alpha-L-fucosyltransferase-like gene, two UDP-glucosyl transferase family protein (Vr36280 and Vr39089), an acidic endochitinase, a rhamnose biosynthetic enzyme 1-like, a soyasaponin III rhamnosyltransferase, two glucan endo-1,3beta-glucosidase-like (Vr43285 and Vr18182), and two extensin-like proteins (Vr58311 and Vr46239) were up-regulated; nine genes including a lignin-forming anionic peroxidase-like, two xyloglucan endotransglucosylase/hydrolase (Vr34550 and Vr39412), two UDPglycosyltransferase 83A1-like (Vr45972 and Vr47846), a beta-glucosidase 47 -like, a cellulose synthase-like protein E1-like, an expansin-like B1-like, and an acetyltransferase gene were down-regulated.

Of these, SOMBRERO-like genes are involved in secondary cell wall biogenesis [58]. BURP domain-containing protein 3-like has polygalacturonase activity that is involved in auxin polar transport and cell wall organization [58]. The gene laccase-3-like is involved in the lignin degradation and detoxification of lignin-derived products [58]. Casparian strip membrane proteins (CASPLs) have recently been shown to form membrane scaffolds and direct the local modification of the cell wall in Arabidopsis thaliana [63]. The gene galactose oxidase-like is involved in lignin degradation pathways. The snakin-1-like protein and acidic endochitinase are members of the glycoside hydrolase $(\mathrm{GH})$ family and potentially participate in cellulose catabolic processes and cell wall loosening [64]. The galactoside 2-alpha-L-fucosyltransferase-like gene is involved in cell wall biogenesis. Extensin-like protein is a member of the leucine-rich repeat (LRR) family protein and serves as structural constituent of the cell wall [65]. Acetyltransferase plays a role in the cutin biosynthetic process [58]. In summary, these results suggest that most of the genes associated with cell wall loosening were strongly induced by IBA at stage 1 , while genes with the potential to be active in cell wall synthesis were repressed by IBA in stage 1 . Conversely, the majority of genes involved in cell wall weakening began to be down-regulated and most of the genes participating in cell wall synthesis were highly up-regulated by IBA in stage 2 . These results suggest that one of the mechanisms by which IBA promotes adventitious rooting is the effective regulation of the expression of genes associated with cell wall loosening and remodeling.

\section{Cell redox homeostasis and stress response}

During stage 1, two glutathione S-transferase (GST) genes (Vr42761 and Vr40194), a cationic peroxidase 1-like gene, a nitronate monooxygenase-like gene, three seed maturation protein LEA 4 genes (Vr18423, Vr12933, and Vr23198), a low-temperature-induced 65-kDa protein-like gene, a cytochrome P450 82A3 gene, a 2-oxoglutarate/ $\mathrm{Fe}(\mathrm{II})$-dependent dioxygenase gene, and a probable 
nucleoredoxin 2-like isoform 2 were up-regulated; four genes encoding lipoxygenases (Vr38595, Vr25042, Vr40522, and Vr43523), a protein IQ-DOMAIN 1-like gene, and a cytochrome P450 monooxygenase CYP93D1 gene were down-regulated. During stage 2, three peroxidase 1-like genes (Vr43362, Vr46509, and Vr40373), a FAD-linked oxidoreductase 1 gene, and a gibberellin 3beta-dioxygenase 3-like gene as well as genes responding to water deprivation such as a lachrymatory-factor synthase-like, a potassium channel AKT1-like, and a DNA-damage-repair/toleration protein DRT100-like precursor gene were up-regulated; five lipoxygenase genes (Vr25042, Vr42442, Vr43510, Vr43523, and Vr38595), an early nodulin-like protein 1-like (electron carrier activity) gene, three peroxidase genes ( Vr46854, Vr45676, and Vr28565), three protein SRG1-like (iron/ascorbate family of oxidoreductases) genes (Vr53443, Vr26676, and Vr13190), and two protein IQ-DOMAIN 1-like genes (Vr12929 and Vr49621) were down-regulated. When stage 2 was compared with stage 1, eight peroxidase genes (Vr43362, Vr41032, Vr42734, Vr48815, Vr59402, Vr67115, $\mathrm{Vr} 31903$, and $\mathrm{Vr}$ 40216) and three cationic peroxidase 1-like genes (Vr43362, Vr46509, and Vr58732) were upregulated; a 2-oxoglutarate/Fe(II)-dependent dioxygenaselike, two cytochrome P450 (Vr37396 and Vr45214), two peroxidase genes (Vr39358 and Vr40642), a GIR1, protein IQ-DOMAIN 14-like, two pleiotropic drug resistance protein 1-like (an $A B C$ transporter $G$ family member) (Vr32712 and Vr48443), and the embryo-abundant protein EMB (S-adenosylmethionine-dependent methyltransferase) were down-regulated.

Glutathione S-transferases (GSTs) play a vital role in the responses to various stresses and were highly induced by IBA [66]. Peroxidase activity has been shown to clearly increase during IBA-induced adventitious rooting [34]. Lachrymatory-factor synthase-like, a pyrabactin resistance 1-like (PYR1) protein, functions as an abscisic acid sensor and mediates its signaling [67]. Lipoxygenases (LOXs) have been shown to be involved in growth, development and oxidative stress [68] and are significantly expressed in tissue cultures for adventitious rooting [22]. In plants, LOXs oxidize linolenic acid present in membranes to result in the lipoperoxidation of membranes $[69,70]$. The cytochrome P450 monooxygenase family of proteins has a variety of functions including anthocyanin accumulation, brassinosteroid biosynthetic processes, cell wall organization, oxidationreduction processes, and indole-containing compound metabolic processes [71]. A member of cytochrome P450 has been shown to regulate adventitious rooting, and a loss of function mutant for the cytochrome P450 CYP83B1, which is involved in the indole glucosinolate pathway, spontaneously produces an excess of adventitious roots [72]. IQ-DOMAIN 1 is a $\mathrm{Ca}^{2+}$-dependent calmodulin-binding protein involved in glucosinolate metabolism in response to biotic challenge [73]. GIR1, a member of the GASA gibberellin regulated cysteine rich protein family, is up-regulated by the plant hormone gibberellin and is involved in defense responses [74]. Late embryogenesis abundant (LEA) proteins act as water-binding molecules, membrane-stabilizers, and ion modulators and are induced by drought stress [50, 75]. Although the changes in gene expression in response to IBA are complex, analysis from our results indicates that antioxidative processes and water deprivation responses were highly up-regulated, while lipoperoxidation of membranes were strongly alleviated by IBA treatment during the early stages of adventitious rooting.

\section{Secondary metabolites and flavonoid and terpenoid biosynthesis}

During stage 1, one flavanone 3-dioxygenase-like gene was up-regulated, while 16 genes, including three flavanone 3-hydroxylase genes (Vr39135, Vr67264, and Vr39068), a beta-amyrin synthase gene, a 3-hydroxy-3methylglutaryl-CoA reductase 1-like gene, an anthocyanin 3'-O-beta-glucosyltransferase-like gene, a ropinone reductase homolog gene, an anthocyanidin synthase gene, a secologanin synthase-like isoform 1 (cytochrome P450) gene, a hydroxymethylglutaryl-CoA synthase-like gene, a UDP-glycose:flavonoid glycosyltransferase gene, a 8hydroxyquercetin 8-O-methyltransferase-like isoform 1 gene, a hydroxyl cinnamoyl-CoA shikimate/quinate hydroxyl cinnamoyl transferase-like gene, a 1-deoxy-D-xylulose-5-phosphate synthase gene (isopentenyl transferase involved in terpenoid biosynthesis), a polyphenol oxidase gene, a cytochrome b5-like gene, and a protein TRANSPARENT TESTA 12-like gene (a flavonoid biosynthesis pathway gene [58]), were down-regulated. During stage 2, an ent-kaurenoic acid oxidase 2-like gene was upregulated, while a 1-deoxy-D-xylulose-5-phosphate synthase gene, a chalcone synthase-like (CHS), and a flavanone 3-hydroxylase gene were down-regulated. When compared with stage 1, a UDP-glycose:flavonoid glycosyltransferase, a hydroxycinnamoyltransferase-like (spermidine hydroxy cinnamoyl transferase), a 3-hydroxy-3-methylglutaryl-CoA reductase 1-like (involved in terpenoid backbone biosynthesis), an ent-kaurenoic acid oxidase 2-like, an 7-ethoxycoumarin O-deethylase-like gene (flavonoid 3'-monooxygenase, has oxidoreductase activity), and a 3'-hydroxy-N-methyl-(S)-coclaurine 4'-Omethyltransferase-like (S-adenosylmethionine-dependent methyltransferases) gene were up-regulated; 11 genes including two isoflavone reductase-like $(\mathrm{NAD}(\mathrm{P}) \mathrm{H}-$ dependent oxidoreductase activity) (Vr34208 and Vr 33951), three isoflavone synthase (Vr43861, Vr50428, and Vr47942), an isoflavone 2'-hydroxylase-like (cytochrome P450), two leucoanthocyanidin dioxygenase-like 
(Vr36176 and Vr44347) (involved in anthocyanincontaining compound biosynthetic process), a flavanone 3-dioxygenase-like, and two isoliquiritigenin 2'-O-methyltransferase-like genes (Vr49125 and Vr47010) were down-regulated.

Flavonoid metabolism exerts roles in development, signaling, and stress responses in plants. Flavonoids were shown to be involved in adventitious rooting [22, 31]. In addition, 8-hydroxyquercetin 8-O-methyltransferase, quinate hydroxyl cinnamoyl transferase, and polyphenol oxidase are also involved in the lignin biosynthetic pathway. The present study indicates that IBA treatment principally down-regulated the expression of genes associated with secondary metabolism, including flavonoid synthesis and lignin biosynthesis, during the early stages of adventitious rooting, especially at root induction stage in mung bean seedlings. During the root primordia formation phase in $P$. contorta, transcripts encoding enzymes of the flavonoid pathway were up-regulated [27]. One of these flavonoid pathway proteins, chalcone synthase, and a pathogenesisrelated protein contribute to a constitutive defense barrier in the root epidermis of the pea [76].

\section{Amino acid and nucleotide metabolism and protein transport and degradation}

During stage 1, two 5'-adenylylsulfate reductase 3 genes (Vr42149 and Vr44003), a CTP synthase-like, and an adenosine deaminase-like gene were up-regulated; three genes related to protein folding and degradation including two trypsin protease inhibitor genes (Vr34971 and Vr35851), a RING-H2 finger protein ATL80-like, and a chaperonin CPN60-like 2 gene were down-regulated. Nutrient reservoir-related genes such as a stem $28-\mathrm{kDa}$ glycoprotein-like gene, a glutelin type-A 2-like gene, and an ATP-citrate synthase alpha chain protein 1-like gene were down-regulated. During stage 2, a prunin 2 precursor, an oligopeptide transporter 1-like, a RING-H2 finger protein ATL60-like, an aspartic proteinase nepenthesin1-like, and a vignain-like gene were up-regulated; two chaperonin CPN60-like 2 genes (Vr40776 and Vr24119), an aspartic proteinase-like, a multicystatin, a stem $28-\mathrm{kDa}$ glycoprotein-like, a probable purine permease 9-like, and a cucumisin-like gene were down-regulated.

When stage 2 was compared with stage 1 , a prunin 2 precursor, a $34-\mathrm{kDa}$ maturing seed protein, an oligopeptide transporter 1-like, a probable amino acid permease 7-like, a probable E3 ubiquitin-protein ligase HERC1like gene, two heat shock 70-kDa protein-like (Vr40796 and Vr42894), and an aspartic proteinase nepenthesin-1like were up-regulated; a germin-like protein 14, a glutamine amidotransferase-like protein, two lysine histidine transporter (Vr55277 and Vr54148), two cysteine protease (Vr38917 Vr38984, and Vr60819), a leurain-like protease, a proton-coupled amino acid transporter, a branched amino acid transaminase, an adenosine deaminase-like protein-like, an aspartic proteinase nepenthesin-1-like, a dnaJ-like protein R260-like, a formate dehydrogenase, an E3 ubiquitin-protein ligase RMA1H1-like, and a probable GMP synthase-like gene were down-regulated.

Of those, 5'-adenylylsulfate reductase 3 reduces sulfate for cysteine biosynthesis involved in cysteine biosynthesis and cell redox homeostasis [58]. Adenosine deaminase-like, which is a putative nucleoside deaminase, may catalyze the hydrolytic deamination of adenosine and play a role in purine metabolism [58]. RING$\mathrm{H} 2$ finger proteins, E3 ubiquitin-protein ligase, and heat shock $70-\mathrm{kDa}$ protein-like are involved in the pathway of protein ubiquitination and in the early steps of the plant defense signaling pathway [58]. Chaperonin CPN60-like 2 may facilitate the correct folding of imported proteins and is implicated in stress response [58]. DnaJ-like protein R260-like also is a chaperone protein, which is required for peroxisomal protein import and maintains the function of peroxisomes [58]. Stem 28-kDa glycoproteinlike, glutelin type-A 2-like, and prunin 2 precursor, and germin-like protein are seed storage proteins. ATP-citrate synthase alpha chain protein 1-like has transferase activity, which transfer acyl groups and is involved in cellular carbohydrate metabolic process [58]. Oligopeptide transporter 1-like is an amino acid/oligopeptide transporter. Aspartic proteinase nepenthesin-1-like, aspartic proteinase-like, vignain-like, cucumisin-like, and 34- $\mathrm{kDa}$ maturing seed protein act as an endopeptidase which is involved in mobilizing storage proteins in seeds and response to water deprivation [58]. GMP synthase-like, also glutamine amidotransferase, is involved in glutamine-hydrolyzing. Multicystatin is a cysteine-type endopeptidase inhibitor, which probably has a role in the plant defense system and is induced by wounding [58]. Formate dehydrogenase and E3 ubiquitin-protein ligase RMA1H1-like also are involved in response to wounding. These results indicate that IBA up-regulated the genes associated with the synthesis and transport of amino acids and nucleotides but down-regulated the genes involved in the protein degradation and processing during early stage of rooting. Furthermore, IBA may alleviate the stresses of wounding and water deprivation occurred at the root initiation stage via up-regulating the expression of the genes associated with protein processing, such as the genes of chaperonin and heat shock 70 .

\section{Lipid and sugar metabolic processes}

A sterol 4-alpha-methyl-oxidase gene and an omega-6 fatty acid desaturase gene were up-regulated, and a patatin-like protein was down-regulated during stage 1 . Four genes coding for patatin group A-3-like (Vr43029, 
Vr58791, Vr22680, and Vr22680) and a carboxylesterase 15 -like gene were down-regulated during stage 2 . When compared with stage 1, two GDSL esterase/lipase CPRD49 genes (Vr13168 and Vr29049), a lipid transfer-like protein, and a bidirectional sugar transporter SWEET3-like gene were up-regulated; a sterol 4-alpha-methyl-oxidase was down-regulated.

Sterol 4-alpha-methyl-oxidase and omega- 6 fatty acid desaturase are involved in fatty acid biosynthetic process [58]. Omega-6 fatty acid desaturase catalyze the biosynthesis of 18:3 fatty acids, which is an important constituent of plant membranes [58]. GDSL esterase/lipase CPRD49 and carboxylesterase 15-like are the members of esterases and lipases, and patatin-like and patatin group A-3-like are the members of phospholipase, which are involved in lipid degradation and plant defense [58]. Obviously, IBA up-regulated the expression of genes associated with fatty acid synthesis but down-regulated the genes for lipid hydrolization during both stages 1 and 2 . Furthermore, IBA up-regulated the genes associated with lipid and sugar transport as well as lipid degradation from stage 1 to stage 2 .

\section{Photosynthesis}

Two genes for LHCII type I chlorophyll a/b-binding proteins (Vr22793 and Vr23315) were down-regulated during stage 1. Four LHCII type I chlorophyll a/b-binding protein genes (Vr38329, Vr22793, Vr38285, and Vr22637), a photosystem II 22-kDa protein, and a chlorophyll a-b binding protein gene were up-regulated during stage 2 compared with stage 1 , suggesting that photosynthetic activity was reduced during stage 1 and then was increased within $24 \mathrm{~h}$ by IBA treatment.

\section{Signal transduction}

A leucine-rich repeat receptor-like protein kinase PXL2like and a membrane-associated kinase regulator 6-like gene were up-regulated during stage 1 . When compared with stage 1, genes encoding a CBL-interacting serine/ threonine-protein kinase 21-like, a leucine-rich repeat receptor-like protein kinase IMK2-like, and two protein suppressor of PHYA-105 1-like genes (Vr26363 and Vr48612) were up-regulated; a membrane-associated kinase regulator 6-like gene was down-regulated.

Leucine-rich repeat receptor-like protein kinase PXL2like, CBL-interacting serine/threonine-protein kinase 21like, leucine-rich repeat receptor-like protein kinase IMK2-like, and membrane-associated kinase regulator 6like belong to the transmembrane receptor protein serine/threonine kinase, which is involved in signaling pathway and cell differentiation [58]. Protein suppressor of PHYA-105 1-like is involved in the phytochrome signaling pathway [58]. The result suggests that IBA mediated in the signaling transduction for promoting adventitious rooting.

\section{Cell cycle}

Genes coding for a syntaxin-related protein KNOLLE-like, three G2/mitotic-specific cyclins (Vr45997, Vr48717, and Vr24479), and an anaphase-promoting complex subunit cdc20-like protein were up-regulated during stage 2 compared with stage 1 . Syntaxin-related protein KNOLLE-like direct movement of proteins in a cell and the membrane organization process [58]. G2/mitotic-specific cyclins are essential for the control of the cell cycle at the G2/M and G1/S (mitosis) transition [57]. Anaphase-promoting complex subunit cdc20-like is implicated in a variety of functions ranging from signal transduction and transcription regulation to cell cycle control [58]. This indicates that cell mitosis started from the stage 2 and IBA promoted this process.

\section{Other genes}

A gene encoding a soluble inorganic pyrophosphataselike protein was down-regulated at stage 2 . When compared with stage 1, a soluble inorganic pyrophosphataselike and a nitrate transporter 1.1-like gene were upregulated; Over-expression of the pyrophosphatase gene increases salt and drought resistance and shoot and root biomass as well as improves nutrient use efficiencies [77]. Nitrate transporter was reported to act as a nitrate sensor that trigger a specific auxin-activated signaling pathway stimulating lateral root growth and may be involved in response to water deprivation $[58,78]$. Clearly, our results may indicate that IBA also modulates the phosphate-containing compound metabolic process and nitrate transport to response to certain stresses and further for adventitious rooting.

\section{Genes involved in plant hormone signaling were significantly regulated by IBA during the adventitious rooting process}

Given that plant hormones are generally considered to be vital modulators of adventitious rooting, we further examined the genes coding for proteins involved in plant hormone signaling transduction during stage 1 and stage 2. A total of 143 unigenes (fold change $>2$ ), including 92 auxin-related genes, 37 ethylene-related genes, five cytokinin-related genes, and eight gibberellin genes, were identified (Additional file 7).

In the group of auxin-related genes, a total of 60 genes, with 52 up-regulated 2.2- to 88 -fold and eight down-regulated 3- to 8-fold, and a total of 41 genes, with 33 up-regulated 2- to 37-fold and seven downregulated 2 - to 11 -fold, were identified at stage 1 and stage 2, respectively. The down-regulated genes at stage 1 included an $\mathrm{ABC}$ transporter, two Aux5NG4-like, a 
PIN4b (auxin efflux carrier), and four IAA-amino acid hydrolase ILR1-like genes; at stage 2, the genes included six Aux5NG4-like genes and an auxin response factor 9like gene. Compared with stage 1, a total of 38 genes, including 11 up-regulated and 37 down-regulated, were detected at stage 2 . The up-regulated genes were primarily members of the $A B C$ transporter $G$ family, while down-regulated genes were mostly members of the AUX family. Auxin efflux carriers control auxin distribution to establish and maintain auxin concentration gradients in various tissues [79], triggering the establishment of new growth axes [80]. The $\mathrm{ABC}$ transporter has been known to function as an auxin carrier complex in cellular auxin efflux and influx [81]. These results indicate that most of the genes related to auxin signaling were significantly up-regulated by IBA treatment. The downregulation of $\mathrm{PIN}$ and the $\mathrm{ABC}$ transporter during stage 1 and up-regulation of the $\mathrm{ABC}$ transporter during stage 2 suggest that auxin transport occurred in stage 2 . In other studies, the expression of PIN1 and AUX were upregulated by IBA treatment and are essential for adventitious root formation [15].

In the group of ethylene-related genes, a total of 17 genes, with 15 up-regulated 2.2- to 42-fold and two down-regulated, and a total of 11 genes, with eight upregulated 2- to 18 -fold and three down-regulated, were identified at stage 1 and stage 2, respectively. Compared with stage 1 , there were a total of 20 genes, with 9 upregulated and 11 down-regulated genes, at stage 2 . Genes coding for an ACS and an ethylene receptor were up-regulated both at stage 1 and stage 2; however, genes coding for an ACC oxidase, an ACS, and three AP2/ ERFs were down-regulated from stage 1 to stage 2 . Ethylene biosynthesis has been demonstrated to be required for adventitious root formation, and there is crosstalk between ethylene and auxin during the process of adventitious root formation [56, 82]. These results suggest that genes associated with ethylene synthesis and signaling were up-regulated by IBA treatment. It appears that IBA-induced ethylene production may be a factor involved in the stimulation of adventitious rooting [11].

Five cytokinin-related genes were detected. Of these, a cytokinin-induced message coding gene was up-regulated both at stage 1 and stage 2, while four genes coding for enzymes involved in cytokinin synthesis were downregulated at stage 1 and from stage 1 to stage 2. Exogenous application of IBA greatly inhibited genes involved in cytokinin biosynthesis [31].

Eight genes involved in the gibberellin (GA) signaling pathway were detected, among which a gibberellic acidstimulated protein 1 and a gibberellin 3-beta-dioxygenase 3-like gene were up-regulated both at stage 1 and stage 2 as well as from stage 1 to stage 2 , and five gibberellin oxidase (GA20ox) genes and a GIR1 gene were downregulated from stage 1 to stage 2 . Both gibberellic acidstimulated protein 1 and GIR1 are the GASA gibberellin regulated cysteine rich proteins, which regulate many plant development processes via cell division and/or elongation and respond to biotic and abiotic stresses [58, 74, 83]. Gibberellin 3-beta-dioxygenase 3-like is involved in gibberellin biosynthesis, which converts the inactive GA precursors GA9 and GA20 in the bioactives GA4 and GA1. GA20ox is a key oxidase enzyme in the biosynthesis of GA that catalyzes the conversion of GA12 and GA53 to GA9 and GA20 respectively [58]. GA treatment has been shown to negatively affect adventitious rooting [84]. This study indicates that IBA treatment inhibited the GA synthesis genes, leading to GA synthesis inhibition.

\section{Transcription factor (TF) encoding genes were significantly regulated by IBA during the process of adventitious rooting}

To further understand the roles of transcription factors in regulating the early stages of rooting, the expression levels of a total of 1,008 TF coding genes that were differentially expressed in the samples were analyzed. Among these, 345 TF genes were significantly expressed with fold-changes $>2$, of which 204 TF genes with 107 up-regulated and 97 down-regulated were detected at stage 1, 199 TF genes with 38 up-regulated and 161 down-regulated were detected at stage 2 , and $204 \mathrm{TF}$ genes with 77 up-regulated and 127 down-regulated were detected in stage 2 versus stage 1 . The majority were from TF families such as auxin-response factors (ARF, 12), indoleacetic acid-induced protein (AUX/IAA, 7), LOB (7), MYB (32), MYC (3), bHLH (31), WRKY (18), NAC (25), APETALA2 and ethylene-responsive element binding proteins (AP2/ERF, 31), HSF (10), homeobox leucine protein (24), zinc finger protein (33), DOF (8), basic leucine zipper (bZIP, 7), C2H2 (3), and E2F (2).

The most differentially expressed TF genes are summarized in Additional file 8. Three NAM family proteins, specifically two protein SOMBRERO-like and a protein FEZ-like, which modulates meristems and primordial development, were up-regulated by approximately 16 - to 32 -fold at stage 1 and stage 2 . In the LOB family, $L O B 16$ and $L O B 29$ were up-regulated approximately 16 to 32-fold both at stage 1 and stage 2, while $L O B 4$ and $L O B 21$ were down-regulated by approximately 2 - to 16 fold both at stage 1 and stage 2 . This result indicates that, in the LOB family, LOB16 and LOB29 were highly up-regulated by IBA treatment. A number of auxinresponsive LOB-domain genes have been found to act early in auxin signaling and regulate adventitious rooting [18]. Of these, the Arabidopsis genes LBD16 and LBD29 were up-regulated by auxin and directly activated by the 
products of the early auxin-responsive genes ARF7 and ARF19 [85, 86]. LBD29 maintains the cell division capacity of the pericycle in response to auxin [87].

The AP2/ERF, MYB, NAC, WRKY, and bHLH families were found to be significantly expressed during early stages of adventitious root formation in poplar, and the MYB and AP2/ERF families were the most highly modulated transcription factors [26]. Among the six AP/ERF genes detected, an ethylene-responsive transcription factor 4-like was up-regulated at stage 1 and five genes were down-regulated at stage 2. AP2/ERFs have been shown to regulate a number of developmental processes [88]. AP2/ERF families were the most highly modulated transcription factors during IBA-induced adventitious rooting $[26,89]$. They have been shown to be key endogenous regulators of adventitious rooting in poplar [90].

In the bHLH family, two genes (Vr34156 and Vr33273) were up-regulated at stage 1 and two genes (Vr44886 and Vr26937) were up-regulated at stage 2 and from stage 1 to stage 2; however, $b H L H 137$ was down-regulated at stage 2 and from stage 1 to stage 2. In the MYB family, MYB134 was down-regulated at stage 1 and stage 2 but upregulated from stage 1 to stage 2. MYB308, MYB363, $M Y B 113$, and MYB114 were down-regulated at stage 1 or stage 2 but up-regulated from stage 1 to stage 2. MYB315 was down-regulated at stage 2 and from stage 1 to stage 2 . In the NAC family, six NAC domain protein coding genes (Vr40604, Vr21172, Vr52381, Vr42870, Vr42963, and Vr50705) were down-regulated at stage 1 and stage 2 with the exception of Vr25638, which was up-regulated from stage 1 to stage 2 . Eight zinc finger protein genes were down-regulated at stage 1 or stage 2 and from stage 1 to stage 2. In addition, a WRKY 2 gene was down-regulated 32-fold at stage 1. A WUSCHEL-related homeobox (WOX) gene, which encodes an auxin-inducible transcription factor that is highly responsive to auxin and is specifically expressed in quiescent center $(\mathrm{QC})$ maintenance and the root apical meristem (RAM) [91], was upregulated at stage 2 , suggesting the occurrence of RAM formation in stage 2 . These results indicate that the majority of bHLH transcription factors were up-regulated, while the majority of MYBs, NACs, zinc finger proteins, and WRKY were down-regulated by IBA treatment during the early stages of adventitious rooting.

\section{Validation of gene expression using qRT-PCR}

To validate the differential expression data obtained through statistical comparison of RPKM values, a total of 36 interesting DEGs of five types were identified: 17 auxin signaling-related genes, 14 stress response-related genes, three $L B D$ genes, and two MYB genes. These genes were selected for validation of the transcriptomic data using real-time quantitative PCR (qRT-PCR). Detailed information on these genes is presented in Additional file 7.
Based on the RNA-Seq results and the study published by Jian et al. [92], we selected three genes, CPY20, eIF5A, and ACTIN (Actin-related protein 4), as internal reference genes for qRT-PCR experiments. The qRT-PCR results showed that $C P Y 20$ was the most stable housekeeping gene, so it was used to calculate the relative expression levels in this study. The expression levels of all the genes measured by qRT-PCR showed a strong correlation to the RNA-Seq data (Fig. 6). The correlation analysis between the RNA-Seq and qRT-PCR data showed that the correlation coefficient of 29 of the 36 data sets is $r>0.938$. During two time points for adventitious rooting, the expression levels of seven ARF family genes exhibited significantly decreasing trends both in the water and IBA treatments, but they were significantly up-regulated by IBA, with the exception of $A R F 3$ and $A R F 19$, compared with the controls. The four IAA (auxin inducible protein) family genes, four $A U X$ family genes, and one PIN gene were significantly up-regulated at $6 \mathrm{~h}$ both in the water and IBA treatments and were significantly induced by IBA. The $L B D 29$ and $L B D 41$ genes were significantly up-regulated by IBA at two time points. The two genes MYB134 and MYB114 exhibited sharp decreases at two time points and a slight increase at $24 \mathrm{~h}$ compared with $6 \mathrm{~h}$, but their expression levels were up-regulated by IBA at $6 \mathrm{~h}$. The four $N A C$ family genes were significantly upregulated by IBA at $6 \mathrm{~h}$ compared with the controls. The genes AHK2 (Arabidopsis histidine kinase) and AHK3 were significantly up-regulated by IBA at two time points. The genes cationic peroxidase 1 (PER1) and PER2 exhibited large increases at two time points but were less affected by IBA treatment. The gene quinone oxidoreductase-like protein $(Q O R L)$ was strongly up-regulated by IBA at $6 \mathrm{~h}$. The ARF transcription factors mediate auxin signaling at the transcriptional level by regulating the expression of auxinresponsive genes. The $L B D$ genes contain at least one auxin-responsive element (AuxRE) and are involved in auxin-mediating development processes. Several members of the LOB-domain transcription factors have been identified and shown to mediate adventitious rooting $[19,22,52]$. Both $L B D 16$ and $L B D 29$ were up-regulated by auxin during adventitious rooting in Arabidopsis hypocotyls and stems [52]. The AUX family genes have also been shown to be essential for adventitious root formation [15]. NAC transcription factors have been reported to contribute to various developmental processes, such as shoot apical meristem (SAM) development [93], lateral root development [94], and secondary wall formation [95]. Moreover, NAC genes are also involved in responses to stress, such as drought and high salinity [96]. AHK1, AHK2, and AHK3 have been identified as cytokinin receptors and are involved in the water stress response during the early vegetative stages of plant growth and regulation of meristem development [97]. Arabidopsis mutants lacking AHK2, AHK3, and AHK4 exhibit enhanced adventitious root growth [98]. The quinone 


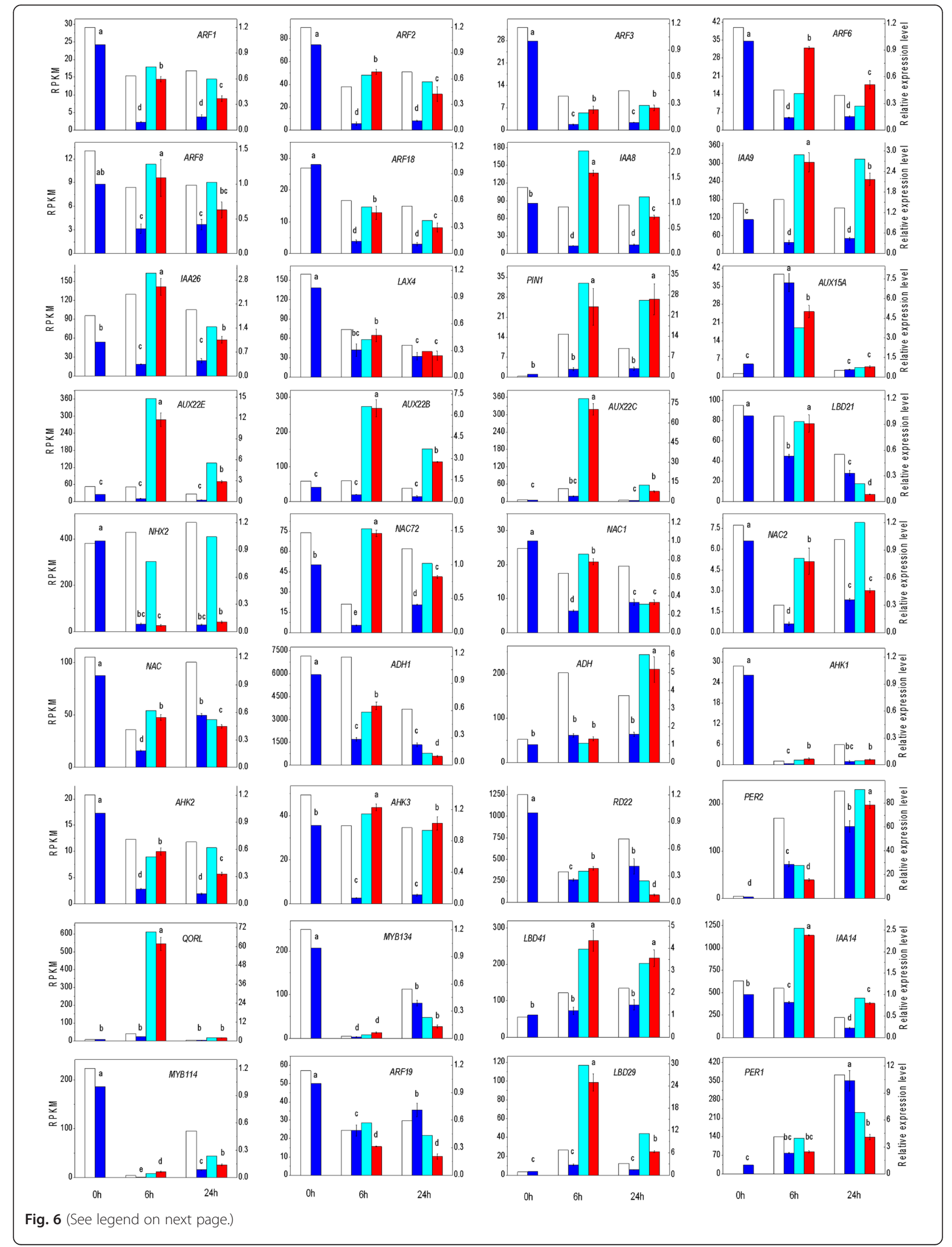


(See figure on previous page.)

Fig. 6 Validation of selected genes involved in adventitious rooting by qRT-PCR. The gene expression levels measured by qRT-PCR were compared with that of RNA-Seq. The data of Con, Wat 6, and Wat 24 in the figure are the same as our previous study published recently [39]. White histograms represent expression levels determined by RNA-Seq in RPKM units (left axis), while gray columns represent gene expression levels determined by qRT-PCR and normalized to control genes (right axis). Bars represent the mean ( \pm SE) of three experiments. Different letters (a, b, and c) represent statistically significant differences $(P<0.01)$ among the qRT-PCR data, analyzed using Student's t-test

oxidoreductase-like protein (QORL), also known as NADH dehydrogenase, which is a zinc-binding dehydrogenase, is involved in the electron transport chain, photorespiration, and redox process. Therefore, our results confirmed that IBA can not only enhance the expression of several auxin signaling control genes such as ARFs, AUX/IAAs, and PINs, as well as several genes from the $L O B$ and $M Y B$ transcription factor families, but can promote the expression of various stress response-related genes such as NAC, AHKs, and QORL to further increase the stress tolerance of the cells, thus leading to efficient adventitious rooting.

\section{Conclusions}

This study provides a comprehensive transcriptome dataset for adventitious rooting in mung bean using the NGS-based Illumina paired-end sequencing platform. A comparative analysis of expression profiles between different rooting developmental time-points as well as between water treatment and IBA treatment yielded subsets of DEGs. Functional categories of significantly enriched GO terms and over-represented biological pathways are presented here. Interestingly, DEG analysis revealed the most significantly and abundantly expressed genes in response to IBA, including genes associated with auxin homeostasis and signaling, ethylene pathway, cytokinin pathway, transcription factors, cell wall modification, cell redox homeostasis and the stress response, secondary metabolites and flavonoid and terpenoid biosynthesis, amino acid and nucleotide metabolism and protein transport and degradation, lipid and sugar metabolic process, photosynthesis, signal transduction, and the cell cycle. Furthermore, a total of 143 DEGs specifically involved in plant hormone signaling and a total of $345 \mathrm{TF}$ genes also responded to IBA. The transcriptomic data reveal that profound cellular and metabolic reorganization occurs during the root induction phase. In conclusion, the transcriptomic data generated in the present study for mung bean adventitious rooting provide insights into the molecular mechanisms that control IBA-induced adventitious rooting.

\section{Materials and methods}

\section{Plant material and culture conditions}

Mung bean [Vigna radiata (L.) R. Wilczek] seeds were washed in distilled water, surface-sterilized in a $6 \%$ $\mathrm{NaClO}$ solution for $15 \mathrm{~min}$ and rinsed three times in sterile distilled water. The seeds were subsequently germinated under a thin layer of sterilized perlite in Petri dishes in a growth chamber at $25 \pm 1{ }^{\circ} \mathrm{C}$ for $36 \mathrm{~h}$ in the dark and then at $25 \pm 1{ }^{\circ} \mathrm{C}$ with a 14-h light/10-h dark photoperiod under white fluorescent lamps (PAR of $100 \mu \mathrm{M} \mathrm{m}^{-2} \mathrm{~s}^{-1}$ ). After five days of growth, seedlings that were $5 \mathrm{~cm}$ in height were used for the experiments. The primary roots of the seedlings were removed from the bases of the hypocotyls, and the resulting seedlings were incubated in $50-\mathrm{mL}$ beakers (10 per beaker), each containing $40 \mathrm{~mL}$ of sterilized distilled water or $10 \mu \mathrm{M}$ IBA for 6 to $24 \mathrm{~h}$ under the same aseptic conditions applied to the seedling culture. The 5-mm-long basal region of each hypocotyl, where adventitious roots develop in vitro, was cut and collected separately at the 0,6 , and $24 \mathrm{~h}$ incubation time points, and the harvested samples were separately designated as Con, Wat6, IBA6, Wat24, and IBA24 (Fig. 1). Three biological replicates, each consisting of ten stem cutting bases, were collected per treatment and time point, immediately frozen in liquid nitrogen and stored at $-80{ }^{\circ} \mathrm{C}$ until further analysis.

\section{Total RNA extraction, cDNA library preparation and Illumina sequencing}

Approximately $50 \mathrm{mg}$ of tissue that was fully ground in liquid nitrogen was mixed with $600 \mu \mathrm{L}$ of buffer Rlysis-P (from kit SK8631, Sangon, Shanghai, China) in a 1.5-mL RNase-free tube for $5 \mathrm{~min}$ in a water bath at $65{ }^{\circ} \mathrm{C}$ to ensure sufficient lysis. Next, $60 \mu \mathrm{L}$ of buffer PCA (from kit SK8631) was added and mixed thoroughly, and the mixture was incubated at $-20{ }^{\circ} \mathrm{C}$ for $3 \mathrm{~min}$. After the mixture was centrifuged at $10,000 \times g$ for $5 \mathrm{~min}$ at $4{ }^{\circ} \mathrm{C}$, an equal volume of cooled phenol chloroform (phenol water) was added to the supernatant, mixed, and then centrifuged at $12,000 \times g$ for $5 \mathrm{~min}$ at $4{ }^{\circ} \mathrm{C}$. An equal volume of cooled chloroform was added to the supernatant and mixed. After the sample was centrifuged at 12,000 $\times$ $g$ for $5 \mathrm{~min}$ at $4{ }^{\circ} \mathrm{C}$, an equal volume of cooled isopropanol was added to the supernatant, shaken gently, and left to precipitate for $10 \mathrm{~min}$. After the sample was centrifuged at $12,000 \times g$ for $20 \mathrm{~min}$ at $4{ }^{\circ} \mathrm{C}$, the pellet was recovered, washed twice with $75 \%$ ethanol, dried for 5 $15 \mathrm{~min}$ at ambient temperature, dissolved in $50 \mu \mathrm{L}$ of RNase-free water, and stored at $-80{ }^{\circ} \mathrm{C}$. The resulting RNA integrity was quantified with RNA Integrity Number (RIN) values of 8.1-9.9 using a 2100 Bioanalyzer (Agilent Technologies, Santa Clara, CA, USA), and RNA 
concentration was determined using a NanoDrop ND1000 Spectrophotometer.

Poly (A) mRNAs were enriched from the equal amounts of total RNA from each sample using Oligo(dT) 25 beads (Invitrogen) according to the manufacturer's protocol. The purified mRNA was fragmented using the Fragment Mix reactive system at $94{ }^{\circ} \mathrm{C}$ for $4 \mathrm{~min}$. The reaction system for first-strand cDNA synthesis was composed of Superscript II reverse transcriptase (Invitrogen), First Strand Master Mix, random hexamer primers, and the fragmented mRNA templates. The reaction conditions were as follows: $25{ }^{\circ} \mathrm{C}$ for $10 \mathrm{~min}, 42{ }^{\circ} \mathrm{C}$ for $50 \mathrm{~min}$, and $70{ }^{\circ} \mathrm{C}$ for $15 \mathrm{~min}$, with a final hold at $4{ }^{\circ} \mathrm{C}$. Second-strand cDNA was synthesized using Second Strand Master Mix (Invitrogen). After synthesis, the dscDNA fragments were purified with Agencourt AMPure XP Beads (Agencourt), and the 3' ends were repaired using End Repair Control, followed by purification with AMPure XP beads. Subsequently, Klenow exo (M0212L, NEB) was used to perform adenylation of the 3' ends of the cDNA fragments. After end repair and A-tailing, Illumina paired-end adapters were ligated to the cDNA fragments using T4 Ligase (Fermentas) and purified twice with AMPure XP Beads. Last, selective PCR was used to enrich and amplify the ligated cDNA for cDNA library construction. The PCR procedure was performed as follows: $98^{\circ} \mathrm{C}$ for $30 \mathrm{~s} ; 15$ cycles of $98{ }^{\circ} \mathrm{C}$ for $10 \mathrm{~s}, 60{ }^{\circ} \mathrm{C}$ for $30 \mathrm{~s}, 72{ }^{\circ} \mathrm{C}$ for $30 \mathrm{~s}$, and $72{ }^{\circ} \mathrm{C}$ for $5 \mathrm{~min}$; holding at $4{ }^{\circ} \mathrm{C}$, followed by purification with AMPure XP beads. The quality and quantity of the cDNA library were confirmed using an Agilent 2100 Bioanalyzer and Qubit 2.0 (Life Technologies). Paired-end sequencing of the constructed cDNA library was performed at Sangon Biotech. Co. Ltd. (Shanghai, China) on an Illumina HiSeq 2000 system (Illumina).

\section{De novo assembly and sequence clustering}

After the raw sequence processing, the clean reads were de novo assembled using the Trinity paired-end assembly method [41]. The assembled sequences were clustered with Chrysalis. The longest sequences that could not be extended on either end within each clustered loci were obtained and defined as unigenes. The unigenes were subjected to similarity alignment against protein and nucleotide sequence databases using BLASTx locally installed BLAST+ v2.2.27 software [99] and MEGABLAST, respectively, at an e-value cut-off of e-5. BLAST annotations were filtered using either subject or query coverage $(>30 \%)$ and sequence identity ( $>50 \%$ for MEGABLAST and $>30 \%$ for BLASTx). The assembled unigenes were deposited in the Transcriptome Shotgun Assembly Sequence Database (http://www.ncbi.nlm.nih.gov/sra/ SRR1653637) at DDBJ/EMBL/GenBank under the sequence read archive SRR 1653637 and the accession numbers GBXO01000001-GBXO01078617.

\section{Mapping reads, expression analysis and DEG confirmation} Due to the lack of a reference sequence, the assembled transcripts were assumed the reference sequence. To compute unigene expression levels, the sequences were aligned against the reference transcript sequences [40, $100,101]$. The expression levels of unigenes were measured by mapping back the number of clean reads to the assembled unigenes using BWA-0.6.2-http://bio-bwa. sourceforge.net/ in the end-to-end alignment mode [102]. The number of clean reads mapped to each unigene was calculated and then normalized to RPKM (reads per kb per million reads) using ERANGE3.1 software [30]. Unigene expression levels were analyzed using the DEGseq R package [51] with the MARS (MA-plotbased method with Random Sampling) model. The DEGs between each pair of samples were screened using the Audic-Claverie algorithm [103] with an FDR threshold of $\leq 0.001$ and an absolute value of $\log 2 \geq 1$. Multiple test corrections for the p-value and FDR were performed with the Benjamini-Hochberg correction [104]. To gain insight into the differential expression of genes specifically induced by IBA at each time point, the analyses were performed between IBA6 and Wat 6 and between IBA24 and Wat24, as well as IBA24 relative to IBA6, and were represented as stage 1 , stage 2 , and stage 1 to stage 2 , respectively.

\section{Functional annotation and classification}

All resulting unigenes were annotated according to their sequence similarity to previously annotated genes. First, the unigenes were aligned to the public protein databases NR, SWISS-PROT, TrEMBL, Pfam, and CDD using BLASTx with similarity set at $>30 \%$ and an Evalue $\leq 1 \mathrm{e}-5$. The KOG (Clusters of Orthologous Groups for eukaryotic complete genomes) and KEGG (Kyoto Encyclopedia of Genes and Genomes) pathway annotations were performed by sequence comparisons against the two databases using BLASTALL and KAAS software (ftp://ftp.ncbi.nih.gov/blast/executables/release/2.2.18/) with an E-value $\leq 1 \mathrm{e}-5$. The resulting blast hits were processed using Blast2GO software (version 2.3.5, http:// www.blast2go.de/) [41] with an E-value threshold of 1e5 to retrieve associated GO terms. WEGO software was used for achievement of GO classification [41]. The results that presented the best alignment were used to identify the sequence direction and to predict the coding regions using BLASTx searches against protein databases, with the priority order of NR, SWISS-PROT, KEGG and KOG if conflicting results were obtained. The ESTScan software [105] was used to analyze the unigenes that did not align to any of the above databases. KEGG mapping was used to determine the metabolic pathways. KEGG pathways were retrieved from the KEGG web server (http://www.genome.jp/kegg/) [106- 
108]. To further enrich the pathway annotations, unigenes were submitted to the KEGG Automatic Annotation Server (KAAS) [43], and the single-directional besthit information method was selected. To identify the enriched pathways, the phyper test was used to measure the relative coverage of the annotated KEGG orthologous groups of a pathway against the transcriptome background, and the pathways with a $\mathrm{p}$-value $\leq 0.05$ were classified as enriched.

\section{Quantitative reverse transcription PCR validation}

To validate the RNA-Seq data, we selected 36 genes that were shown to be involved in adventitious root development for q-PCR analysis. PCR samples were harvested from the hypocotyls of three biological replicates under the same conditions as the samples subjected to Illumina sequencing. Total RNA was extracted using TRIzol reagent (Invitrogen, Carlsbad, CA, USA) and purified on RNeasy mini spin columns (Qiagen) with on-column DNase I treatment according to the manufacturer's protocol. RNA integrity was examined with an Agilent Bioanalyzer 2100 (Agilent Technologies). First-strand cDNA was synthesized using an AMV First Strand cDNA Synthesis Kit (Roche Applied Science, Mannheim, Germany) according to the manufacturer's instructions. The gene-specific primer pairs were designed using Primer Premier 5.0 software (Applied Biosystems, Foster City, CA, USA) according to the confirmed sequences (Additional file 9). Real-time PCR was run using a LightCycler 480 II (Roche Applied Science) and ABI SYBR Green PCR Master Mix (ABI, Foster, USA). Reactions were subjected to the following conditions: $95{ }^{\circ} \mathrm{C}$ for $3 \mathrm{~min}$ followed by 40 cycles of $95{ }^{\circ} \mathrm{C}$ for $15 \mathrm{~s}$ and $60{ }^{\circ} \mathrm{C}$ for $40 \mathrm{~s}$. A melting curve analysis was conducted to evaluate the primer specificity for each primer set to verify the presence of a single melting peak after amplification. 'No cDNA' samples (water) and 'no RT' samples were included as negative controls. All reactions were performed with three independent biological replicates, and the expression levels calculated for each sample were based on three technical replicates. Output data were generated with Sequence Detector version 1.3.1 software $(\mathrm{ABI})$. The relative expression levels of the selected genes were calculated in relation to the reference gene using the comparative threshold cycle method with the delta-delta Ct method [109]. Statistical analyses were conducted with Student's t-tests at the $P<0.05$ level of significance. The expression levels of all the genes measured by qRT-PCR showed a strong correlation to the RNA-Seq data.

\section{Availability of data and materials}

The unigene sequence information of this article is available in the Transcriptome Shotgun Assembly Sequence
Database (http://www.ncbi.nlm.nih.gov/sra/SRR1653637) at DDBJ/EMBL/GenBank under the sequence read archive SRR 1653637 and the accession numbers GBXO01000001GBXO01078617. The datasets supporting the conclusions of this article are included within the article and its additional files.

\section{Additional files}

Additional file 1: Sequencing data information. (DOCX $13 \mathrm{~kb}$ ) Additional file 2: Top list of significantly up- and down-regulated GOs. (XLSX $43 \mathrm{~kb}$ )

Additional file 3: Top list of significantly up- and down-regulated KOs. (XLSX $14 \mathrm{~kb}$ )

Additional file 4: Top up- and down regulated DEGs in the sample treated with IBA for $6 \mathbf{h}$. (XLSX $18 \mathrm{~kb})$

Additional file 5: Top up- and down regulated DEGs in the sample treated with IBA for $\mathbf{2 4}$ h. (XLSX $19 \mathrm{~kb}$ )

Additional file 6: Top up- and down regulated DEGs in IBA24 versus IBA6. (XLSX $28 \mathrm{~kb}$ )

Additional file 7: Top significantly up- and down- regulated DEGs involved in plant hormone signaling between the samples. (XLSX $16 \mathrm{~kb}$ )

Additional file 8: Top most differentially expressed transcription factor (TF) coding genes between the samples. (XLSX $12 \mathrm{~kb}$ )

Additional file 9: List of the genes and primers selected for q-PCR validation. (DOCX $22 \mathrm{~kb}$ )

\section{Abbreviations}

ABCB/PGP: ATP binding cassette-type B/ P-Glycoproteins; ACC: 1Aminocyclopropane-1-carboxylic acid; ACO: 1-Aminocyclopropane-1carboxylic acid oxidase; ACS: 1-Aminocyclopropane-1-carboxylic acid synthase; AHK: Arabidopsis histidine kinase; AP2/ERF: Apetala2/Ethylene response factor; ARF: Auxin response factor; ARL1: Adventitious rootiless1; AUX1/LAX: Auxin/IAA; bHLH: Basic/helix-loop-helix; bZIP: basic leucine zipper; CASPL: Casparian strip membrane protein; CHS: Chalcone synthase;

CRL1: Crown rootless1; DEGs: Differentially expressed genes; GH: Glycoside hydrolase; GH3: Gretchen hagen 3; GO: Gene ontology; GST: Glutathione S-transferase; IBA: Indole-3-butyric acid; JA: Jasmonic acid; KAAS: Automatic annotation server; KEGG: Kyoto encyclopedia of genes and genomes; KOG: Clusters of orthologous groups for eukaryotic complete genomes; LBD16: Lateral organ boundaries-domain; LEA: Late embryogenesis abundant; LOB-domain: Lateral organ boundaries-domain; LOXs: Lipoxygenases; LRR: Leucine-rich repeat; NAC: NAM, ATAF, and CUC2; NAM: No apical meristem; NR: NCBI non-redundant protein; PER: Peroxidase; PIN: PIN-formed; PYR: Pyrabactin resistance; QC: Quiescent center; QORL: Quinone oxidoreductase-like protein; qRT-PCR: Real-time quantitative polymerase chain reaction; RAM: Root apical meristem; RNA-seq: RNA-sequencing; RPKM: Reads per kb per million reads; RTCS: Rootless concerning crown and seminal roots; SAM: S-adenosylmethionine; SCR: Scarecrow; WRKY: WRKYGQK domain protein.

Competing interests

The authors declare that they have no competing interests.

\section{Authors' contributions}

LSW conceived of the study, participated in the design of experiment and analysis of the sequence data, and drafted the manuscript. SRF and LY participated in the experiments and performed the statistical analysis. ZY participated in the statistical analysis. All authors read and approved the final manuscript.

\section{Acknowledgements}

This work was supported by the National Natural Science Foundation of China (31560121 and 31260090). 


\section{Author details}

'School of Environmental and Municipal Engineering, Key Laboratory of Extreme Environmental Microbial Resources and Engineering Gansu Province, Lanzhou Jiaotong University, 88 West Anning Road, Lanzhou 730070, P. R. China. ${ }^{2}$ School of Chemical and Biological Engineering, Lanzhou Jiaotong University, 88 West Anning Road, Lanzhou 730070, P.R. China.

\section{Received: 15 July 2015 Accepted: 6 January 2016}

\section{Published online: 12 January 2016}

\section{References}

1. Pacurar DI, Perrone I, Bellini C. Auxin is a central player in the hormone cross-talks that control adventitious rooting. Physiol Plant. 2014;151:83-96.

2. Ramirez-Carvajal GA, Davis JM. Cutting to the base identifying regulators of adventitious rooting. Plant Sig Beh. 2010;5:281-3.

3. Li SW, Xue L, Xu S, Feng H, An L. Mediators, genes and signaling in adventitious rooting. Bot Rev. 2009;75:230-47.

4. Wiesmann Z, Riov J, Epstein E. Comparison of movement and metabolism of indole-3-acetic acid and indole-3-butyric acid in mung bean cuttings. Physiol Plant. 1988;74:556-60.

5. De Klerk GJ, Krieken WVD, Jong JCD. The formation of adventitious roots: new concepts, new possibilities. In Vitro Cell Dev Biol Plant. 1999:35:189-99.

6. Sorin C, Negroni L, Balliau T, Corti H, Jacquemot MP, Davanture M, et al. Proteomic analysis of different mutant genotypes of Arabidopsis led to the identification of 11 proteins correlating with adventitious root development. Plant Physiol. 2006;140:349-64.

7. Ludwig-Müller J, Vertocnik A, Town CD. Analysis of indole-3-butyric acidinduced adventitious root formation on Arabidopsis stem segments. J Exp Bot. 2005;56:2095-105

8. Rout GR. Effect of auxins on adventitious root development from single node cuttings of Camellia sinensis (L.) Kuntze and associated biochemical changes. Plant Growth Regul. 2006:48:111-7.

9. Riov J, Yang SF. Ethylene and ethylene-auxin interaction in adventitious root formation in mung bean (Vigna radiata) cuttings. J Plant Growth Regul. 1989:8:131-41

10. Abel S, Nguyen MD, Chow W. T heologis A. ACS4, a primary indoleacetic acid-responsive gene encoding 1-aminocyclopropane-1-carboxylate synthase in Arabidopsis thaliana - characterization, expression in E. coli, and expression characteristics in response to auxin. J Bio Chem. 1995; 270:19093-9.

11. Pan $\mathrm{R}$, Wang J, Tian $\mathrm{X}$. Influence of ethylene on adventitious root formation in mung bean hypocotyl cuttings. Plant Grow Regul. 2002;36:135-9.

12. Gutierrez L, Bussell JD, Pacurar DI, Schwambach J, Pacurar M, Bellini $C$ Phenotypic plasticity of adventitious rooting in Arabidopsis is controlled by complex regulation of AUXIN RESPONSE FACTOR transcripts and microRNA abundance. Plant Cell. 2009:21:3119-32.

13. Gutierrez L, Mongelard G, Floková K, Păcurar DI, Novák O, Staswick P, et al. Auxin controls Arabidopsis adventitious root initiation by regulating jasmonic acid homeostasis. Plant Cell. 2012;24:2515-27.

14. Mashiguchi K, Tanaka K, Sakai T, Sugawara S, Kawaide H, Natsume M, et al. The main auxin biosynthesis pathway in Arabidopsis. Proc Natl Acad Sci U S A. 2011:108:18512-7.

15. Li Y-H, Zou M-H, Feng B-H, Huang X, Zhang Z, Sun G-M. Molecular cloning and characterization of the genes encoding an auxin efflux carrier and the auxin influx carriers associated with the adventitious root formation in mango (Mangifera indica L.) cotyledon segments. Plant Physiol Biochem. 2012;55:33-42

16. Péret $B$, Swarup $K$, Ferguson $A$, Seth $M$, Yang $Y$, Dhondt $S$, et al. AUX LAX genes encode a family of auxin influx transporters that perform distinct functions during Arabidopsis development. Plant Cell. 2012;24:2874-85.

17. Sukumar P, Maloney GS, Muday GK. Localized induction of the ATP-Binding cassette B19 auxin transporter enhances adventitious root formation in Arabidopsis. Plant Physiol. 2013;162:1392-405.

18. Hochholdinger F, Zimmermann R. Conserved and diverse mechanisms in root development. Curr Opin Plant Biol. 2008;11:70-4.

19. Liu H, Wang S, Yu X, Yu J, He X, Zhang S, et al. ARL1, a LOB-domain protein required for adventitious root formation in rice. Plant J. 2005:43:47-56.

20. Inukai $Y$, Sakamoto T, Ueguchi-Tanaka M, Shibata Y. Crown rootless1, which is essential for crown root formation in rice, is a target of an AUXIN RESPONSE FACTOR in auxin signaling. Plant Cell. 2005;17:1387-96.
21. Taramino G, Sauer M, Stauffer JL, Multani D, Niu X, Sakai H, et al. The maize, Zea mays L. RTCS gene encodes a LOB domain protein that is a key regulator of embryonic seminal and post-embryonic shoot-borne root initiation. Plant J. 2007:50:649-59.

22. Holmes P, Djordjevic MA, Imin N. Global gene expression analysis of in vitro root formation in Medicago truncatula. Fun Plant Biol. 2010;37:1117-31.

23. Shuai B, Reynaga CG, Springer PS. The LATERAL ORGAN BOUNDARIES gene defines a novel, plant-specific gene family. Plant Physiol. 2002;129:747-61.

24. Sánchez C, Vielba JM, Ferro E, Covelo G, Solé A, Abarca D, et al. Two SCARECROW-LIKE genes are induced in response to exogenous auxin in rooting-competent cuttings of distantly related forest species. Tree Physiol. 2007;27:1459-70.

25. Ramirez-Carvajal GA, Morse AM, Dervinis C, Davis JM. The cytokinin type-B response regulator PtRR13 is a negative regulator of adventitious root development in Populus. Plant Physiol. 2009:150:759-71.

26. Rigal A, Yordanov YS, Perrone I, Karlberg A, Tisserant E, Bellini C, et al. The AINTEGUMENTA LIKE1 homeotic transcription factor PtAIL1 controls the formation of adventitious root primordia in poplar. Plant Physiol. 2012;160: 1996-2006.

27. Brinker M, van Zyl L, Liu W, Craig D, Sederoff RR, Clapham DH, et al. Microarray analyses of gene expression during adventitious root development in Pinus contorta. Plant Physiol. 2004;135:1526-39.

28. Annadurai RS, Jayakumar V, Mugasimangalam RC, Katta M. AVSK, Anand S, Gopinathan S, et al. Next generation sequencing and de novo transcriptome analysis of Costus pictus D. Don, a non-model plant with potent anti-diabetic properties. BMC Genomics. 2012;13:663.

29. Huang $\mathrm{H}-\mathrm{H}, \mathrm{Xu} \mathrm{L}-\mathrm{L}$, Tong Z-K, Lin E-P, Liu Q-P, Cheng L-J, et al. De novo characterization of the Chinese fir (Cunninghamia lanceolata) transcriptome and analysis of candidate genes involved in cellulose and lignin biosynthesis. BMC Genomics. 2012;13:648

30. Mortazavi A, Williams BA, McCue K, Schaeffer L, Wold B. Mapping and quantifying mammalian transcriptomes by RNA-Seq. Nat Methods. 2008;5:621-8

31. Wei K, Wang L-Y, Wu L-Y, Zhang C-C, Li H-L, Tan L-O, et al. Transcriptome analysis of indole-3-butyric acid-induced adventitious root formation in nodal cuttings of Camellia sinensis (L.). PLoS One. 2014;9:e107201.

32. Schafleitner R, Nair RM, Rathore A, Wang Y-W, Lin C-Y, Chu S-U, et al. The AVRDC - The World Vegetable Center mungbean (Vigna radiata) core and mini core collections. BMC Genomics. 2015;16:344.

33. Wiesman Z, Riov J, Epstein E. Characterization and rooting ability of indole3-butyric acid conjugates formed during rooting of mung bean cuttings. Plant Physiol. 1989;91:1080-4.

34. Li S-W, Xue L, Xu S, Feng H, An L. IBA-induced changes in antioxidant enzymes during adventitious rooting in mung bean seedlings: the role of $\mathrm{H}_{2} \mathrm{O}_{2}$. Environ Exp Bot. 2009;66:442-50.

35. Batish DR, Singh HP, Kaur S, Kohli RK, Yadav SS. Caffeic acid affects early growth, and morphogenetic response of hypocotyl cuttings of mung bean (Phaseolus aureus). J Plant Physiol. 2008;165:297-305.

36. Yang W, Zhu C, Ma X, Li G, Gan L, Ng D, et al. Hydrogen peroxide is a second messenger in the salicylic acid-triggered adventitious rooting process in mung bean seedlings. PLoS One. 2013;8:e84580.

37. Konishi M, Sugiyama M. Genetic analysis of adventitious root formation with a novel series of temperature-sensitive mutants of Arabidopsis thaliana. Development. 2003;130:5637-47

38. Li S-W, Xue L, Xu S, Feng H, An L. Hydrogen peroxide acts as a signal molecule in the adventitious root formation of mung bean seedlings. Environ Exp Bot. 2009:65:63-71.

39. Li S-W, Shi R-F, Leng Y. De novo characterization of the mung bean transcriptome and transcriptomic analysis of adventitious rooting in seedlings using RNA-Seq. PLoS One. 2015;10:e0132969.

40. Grabherr MG, Haas BJ, Yassour M, Levin JZ, Thompson DA, Amit I, et al. Full-length transcriptome assembly from RNA-Seq data without a reference genome. Nat Biotechnol. 2011;29:644-52.

41. Conesa A, Gotz S, García-Gómez JM, Terol J, Talón M, Robles M. Blast2GO: a universal tool for annotation, visualization and analysis in functional genomics research. Bioinformatics. 2005:21:3674-6.

42. Ye J, Fang $L$, Zheng $H$, Zhang $Y$, Chen J, Zhang Z, et al. WEGO: a web tool for plotting GO annotations. Nucleic Acids Res. 2006;34:W293-7.

43. Moriya Y, Itoh M, Okuda S, Yoshizawa AC, Kanehisa M. KAAS: an automatic genome annotation and pathway reconstruction server. Nucleic Acids Res. 2007;35 suppl 2:W182-5. 
44. Abu-Abied M, Szwerdszarf D, Mordehaev I, Levy A, Rogovoy O, Belausov E, et al. Microarray analysis revealed upregulation of nitrate reductase in juvenile cuttings of Eucalyptus grandis, which correlated with increased nitric oxide production and adventitious root formation. Plant J. 2012;71:787-99.

45. Dixon RA, Achnine L, Kota P, Liu CJ, Reddy MSS, Wang L. The phenylpropanoid pathway and plant defence-a genomics perspective. Mol Plant Pathol. 2002;3:371-90.

46. Davin LB, Jourdes M, Patten AM, Kim KW, Vassao DG, Lewis NG. Dissection of lignin macromolecular configuration and assembly: Comparison to related biochemical processes in allyl/propenyl phenol and lignan biosynthesis. Nat Prod Rep. 2008;25:1015-90.

47. Boudet AM, LaPierre C, Grima-Pettenati J. Biochemistry and molecular biology of lignification. New Phytol. 1995;129:203-36.

48. Tamagnone L, Merida A, Stacey N, Plaskitt K, Parr A, Chang CF, et al. Inhibition of phenolic acid metabolism results in precocious cell death and altered cell morphology in leaves of transgenic tobacco plants. Plant Cell. 1998;10:1801-16

49. Macedo ES, Sircar D, Cardoso HG, Peixe A, Arnholdt-Schmitt B. Involvement of alternative oxidase (AOX) in adventitious rooting of Olea europaea $\mathrm{L}$. microshoots is linked to adaptive phenylpropanoid and lignin metabolism. Plant Cell Rep. 2012;31:1581-90.

50. Alvarez S, Marsh EL, Schroeder SG, Schachtman DP. Metabolomic and proteomic changes in the xylem sap of maize under drought. Plant, Cell Environ. 2008;31:325-40

51. Wang L, Feng Z, Wang X, Wang X, Zhang X. DEGseq: an R package for identifying differentially expressed genes from RNA-Seq data. Bioinformatics. 2010;26:136-8.

52. Welander M, Geier T, Smolka A, Ahlman A, Fan J, Zhu L-H. Orign, timeing, and gene expression profile of adventitious rooting in Arabicopsis hypocotyls and stems. Am J Bot. 2014;101:255-66.

53. Robert S, Kleine-Vehn J, Barbez E, Sauer M, Paciorek T, Baster P, et al. ABP1 mediates auxin inhibition of clathrin-dependent endocytosis in Arabidopsis. Cell. 2010;143:111-21.

54. Quan J, Zhang S, Zhang C, Meng S, Zhao Z, et al. Molecular cloning, characterization and expression analysis of the SAMS gene during adventitious root development in IBA-induced tetraploid black locust. PLoS One. 2014;9:e108709.

55. Negi S, Ivanchenko MG, Muday GK. Ethylene regulates lateral root formation and auxin transport in Arabidopsis thaliana. Plant J. 2008;55:175-87.

56. Negi S, Sukumar P, Liu X, Cohen JD, Muday GK. Genetic dissection of the role of ethylene in regulating auxin-dependent lateral and adventitious root formation in tomato. Plant J. 2010;61:3-15.

57. Stepanova AN, Yun J, Likhacheva AV, Alonso JM. Multilevel interactions between ethylene and auxin in Arabidopsis roots. Plant Cell. 2007;19:2169-85.

58. Swiss-Prot Database. http://www.uniprot.org/. Accessed Aug 2013

59. Solé A, Sánchez C, Vielba JM, Valladares S, Abarca D, Díaz-Sala C Characterization and expression of a Pinus radiata putative ortholog to the Arabidopsis SHORT-ROOT gene. Tree Physiol. 2008;28:1629-39.

60. Vielba JM, Díaz-Sala C, Ferro E, Rico S, Lamprecht M, Abarca D, et al CSSCL1 is differentially regulated upon maturation in chestnut microshoots and is specifically expressed in rooting-competent cells. Tree Physiol. 2011;31:1152-60.

61. Hutchison KW, Singer PB, McInnis S, Diaz-Sala C, Greenwood MS. Expansins are conserved in conifers and expressed in hypocotyls in response to exogenous auxin. Plant Physiol. 1999;120:827-31.

62. Quiroga M, Guerrero C, Botella MA, Barceló A, Amaya I, Medina MI, et al. A tomato peroxidase involved in the synthesis of lignin and suberin. Plant Physiol. 2000;122:1119-27.

63. Roppolo D, Boeckmann B, Pfister A, Boutet E, Rubio MC, Dénervaud-Tendon $\checkmark$, et al. Functional and evolutionary analysis of the CASPARIAN STRIP MEMBRANE DOMAIN PROTEIN family. Plant Physiol. 2014;165:1709-22.

64. Cosgrove DJ. Growth of the plant cell wall. Nat Rev Mol Cell Biol. 2005:6:850-61.

65. Lewis DR, Olex AL, Lundy SR, Turkett WH, Fetrow JS, et al. A kinetic analysis of the auxin transcriptome reveals cell wall remodeling proteins that modulate lateral root development in Arabidopsis. Plant Cell. 2013;25:3329-46.

66. Jiang HW, Liu MJ, Chen IC, Huang CH, Chao LY, et al. A glutathione Stransferase regulated by light and hormones participates in the modulation of Arabidopsis seedling development. Plant Physiol. 2010;154:1646-58.
67. Nishimura N, Sarkeshik A, Nito K, Park SY, Wang A, Carvalho PC, et al. PYR/ PYL/RCAR family members are major in-vivo ABI1 protein phosphatase 2C-interacting proteins in Arabidopsis. Plant J. 2010;61:290-9.

68. Keunen E, Remans T, Opdenakker K, Jozefczak M, Gielen H, Guisez Y, et al. A mutant of the Arabidopsis thaliana LIPOXYGENASE1 gene shows altered signalling and oxidative stress related responses after cadmium exposure. Plant Physiol Biochem. 2013;63:272-80.

69. Maccarrone M, Melino G, Finazzi-Agro A. Lipoxygenases and their involvement in programmed cell death. Cell Death Differ. 2001;8:776-84.

70. Veronico P, Giannino D, Melillo MT, Leone A, Reyes A, Kennedy MW, et al. A novel lipoxygenase in pea roots. Its function in wounding and biotic stress. Plant Physiol. 2006;141:1045-55.

71. Ohnishi T, Godza B, Watanabe B, Fujioka S, Hategan L, Ide K, et al. CYP90A1/ CPD, a brassinosteroid biosynthetic cytochrome P450 of Arabidopsis, catalyzes C-3 oxidation. J Biol Chem. 2012;287:31551-60.

72. Delarue M, Prinsen E, Vanonckelen H, Caboche M, Bellini C. Sur2 mutations of Arabidopsis thaliana define a new locus involved in the control of auxin homeostasis. Plant J. 1998;14:603-11.

73. Buerstenbinder K, Savchenko T, Mueller J, Adamson AW, Stamm G, Kwong $\mathrm{R}$, et al. Arabidopsis calmodulin-binding IQD1 localizes to microtubules and interacts with kinesin light chain-related protein-1. J Biol Chem. 2013;288:1871-82

74. Cao D, Cheng H, Wu W, Soo HM, Peng J. Gibberellin mobilizes distinct DELLA-dependent transcriptomes to regulate seed germination and floral development in Arabidopsis. Plant Physiol. 2006;142:509-25.

75. Hand SC, Menze MA, Toner M, Boswell L, Moore D. LEA proteins during water stress: not just for plants anymore. Annu Rev Physiol. 2011;73:115-34.

76. Mylona P, Moerman M, Yang WC, Gloudemans T, Van de Kerckhove J, van Kammen A, et al. The root epidermis specific pea gene $R H 2$ is homologous to a pathogenesis-related gene. Plant Mol Biol. 1994;26:39-50.

77. Pizzio GA, Paez-Valencia J, Khadilkar AS, Regmi KC, Patron-Soberano A, Zhang $S$, et al. Arabidopsis proton-pumping pyrophosphatase AVP1 expresses strongly in phloem where it is required for PPi metabolism and photosynthate partitioning. Plant Physiol. 2015;167:1541-53.

78. Krapp A, David LC, Chardin C, Girin T, Marmagne A, Leprince AS, et al. Nitrate transport and signalling in Arabidopsis. J Exp Bot. 2014;65:789-98.

79. Zazimalova E, Krecek P, Skupa P, Hoyerova K, Petrasek J. Polar transport of the plant hormone auxin - the role of PIN-FORMED (PIN) proteins. Cell Mol Life Sci. 2007:64:1621-37.

80. Chapman EJ, Estelle M. Mechanism of auxin-regulated gene expression in plants. Annu Rev Genet. 2009;43:265-85.

81. Cho M, Lee SH, Cho H-T. P-glycoprotein4 displays auxin efflux transporter-like action in Arabidopsis root hair cells and tobacco cells. Plant Cell. 2007;19:3930-43.

82. Vidoz ML, Loreti E, Mensuali A, Alpi A, Perata P. Hormonal interplay during adventitious root formation in flooded tomato plants. Plant J. 2010;63:551-62.

83. Li KL, Bai X, Li Y, Cai H, Ji W, Tang LL, et al. GsGASA1 mediated root growth inhibition in response to chronic cold stress is marked by the accumulation of DELLAs. J Plant Physiol. 2011;168:2153-60.

84. Mauriat M, Petterle A, Bellini C, Moritz T. Gibberellins inhibit adventitious rooting in hybrid aspen and Arabidopsis by affecting auxin transport. Plant J. 2014;78:372-84.

85. Okushima Y, Fukaki $\mathrm{H}$, Onoda M, Theologis A, Tasaka M. ARF7 and ARF19 regulate lateral root formation via direct activation of $L B D / A S L$ genes in Arabidopsis. Plant Cell. 2007:19:118-30.

86. Lee HW, Kim NY, Lee DJ, Kim J. LBD18/ASL20 regulates lateral root formation in combination with LBD16/ASL18 downstream of ARF7 and ARF19 in Arabidopsis. Plant Physiol. 2009;151:1377-89.

87. Feng C, Chen M, Xu C-J, Bai L, Yin X-R, Li X, et al. Transcriptomic analysis of Chinese bayberry (Myrica rubra) fruit development and ripening using RNASeq. BMC Genomics. 2012;13:19.

88. Imin N, Nizamidin M, Wu T, Rolfe BG. Factors involved in root formation in Medicago truncatula. J Exp Bot. 2007;58:439-51.

89. Trupiano D, Yordanov Y, Regan S, Meilan R, Tschaplinski T, Scippa GS, et al. Identification, characterization of an AP2/ERF transcription factor that promotes adventitious, lateral root formation in Populus. Planta. 2013;238:271-82.

90. Hatzilazarou SP, Syros TD, Yupsanis TA, Bosabalidis AM, Economou AS. Peroxidases, lignin and anatomy during in vitro and ex vitro rooting of gardenia (Gardenia jasminoides Ellis) microshoots. J Plant Physiol. 2006;163:827-36 
91. Sarkar AK, Luijten M, Miyashima S, Lenhard M, Hashimoto T, Nakajima K, et al. Conserved factors regulate signalling in Arabidopsis thaliana shoot and root stem cell organizers. Nature. 2007:446:811-4.

92. Jian B, Liu B, Bi Y, Hou W, Wu C, Han T. Validation of internal control for gene expression study in soybean by quantitative real-time PCR. BMC Mol Biol. 2008;9:59.

93. Souer E, Van AH, Kloos D, Mol J, Koes R. The No Apical Meristem gene of petunia is required for pattern formation in embryos and flowers and is expressed at meristem and primordia boundaries. Cell. 1996;85:159-70.

94. He XJ, Mu RL, Cao WH, Zhang ZG, Zhang JS, Chen SY. AtNAC2, a transcription factor downstream of ethylene and auxin signaling pathways, is involved in salt stress response and lateral root development. Plant J. 2005:44:903-16.

95. Mitsuda N, Iwase A, Yamamoto H, Yoshida M, Seki M, Shinozaki K, et al. NAC transcription factors, NST1 and NST3, are key regulators of the formation of secondary walls in woody tissues of Arabidopsis. Plant Cell. 2007;19:270-80.

96. Hu H, Dai M, Yao J, Xiao B, Li X, Zhang Q, et al. Overexpressing a NAM, ATAF, and CUC (NAC) transcription factor enhances drought resistance and salt tolerance in rice. Proc Natl Acad Sci U S A. 2006;103:12987-92.

97. Wohlbach DJ, Quirino BF, Sussman MR. Analysis of the Arabidopsis histidine kinase ATHK1 reveals a connection between vegetative osmotic stress sensing and seed maturation. Plant Cell. 2008:20:1101-17.

98. Nishimura C, Ohashi Y, Sato S, Kato T, Tabata S, Ueguchi C. Histidine kinase homologs that act as cytokinin receptors possess overlapping functions in the regulation of shoot and root growth in Arabidopsis. Plant Cell. 2004;16:1365-77.

99. Camacho C, Coulouris G, Avagyan V, Ma N, Papadopoulos J, Bealer K, et al. BLAST+: architecture and applications. BMC Bioinformatics. 2009;10:421.

100. Xu DL, Long H, Liang JJ, Zhang J, Chen X, Li JL, et al. De novo assembly and characterization of the root transcriptome of Aegilops variabilis during an interaction with the cereal cyst nematode. BMC Genomics. 2012;13:133.

101. Barrero RA, Chapman B, Yang Y, Moolhuijzen P, Keeble-Gagnère G, Zhang N et al. De novo assembly of Euphorbia fischeriana root transcriptome identifies prostratin pathway related genes. BMC Genomics. 2011;12:600.

102. Langmead B, Salzberg SL. Fast gapped-read alignment with bowtie 2. Nat Methods. 2012;9:357-9.

103. Audic S, Claverie JM. The significance of digital gene expression profiles. Genome Res. 1997;7:986-95.

104. Benjamini Y, Hochberg Y. Controlling the false discovery rate: a practical and powerful approach to multiple testing. J Roy Stat So Ser B. 1995;57:289-300

105. Iseli C, Jongeneel CV, Bucher P. ESTScan: a program for detecting, evaluating, and reconstructing potential coding regions in EST sequences, Proceedings-International Conference on Intelligent Systems for Molecular Biology. 1999;138-148

106. Kanehisa M, Goto S. KEGG: Kyoto Encyclopedia of Genes and Genomes. Nucleic Acids Res. 2000;28:27-30.

107. Kanehisa M, Goto S, Kawashima S, Okuno Y, Hattori M. The KEGG resource for deciphering the genome. Nucleic Acids Res. 2004;32:D277-80.

108. Mao X, Cai T, Olyarchuk JG, Wei L. Automated genome annotation and pathway identification using the KEGG Orthology (KO) as a controlled vocabulary. Bioinformatics. 2005;21:3787-93.

109. Livak KJ, Schmittgen TD. Analysis of relative gene expression data using real time quantitative PCR and the $2^{-\Delta \Delta C t}$ method. Methods. 2001;25:402-8.

\section{Submit your next manuscript to BioMed Central and we will help you at every step:}

- We accept pre-submission inquiries

- Our selector tool helps you to find the most relevant journal

- We provide round the clock customer support

- Convenient online submission

- Thorough peer review

- Inclusion in PubMed and all major indexing services

- Maximum visibility for your research

Submit your manuscript at www.biomedcentral.com/submit

) Biomed Central 